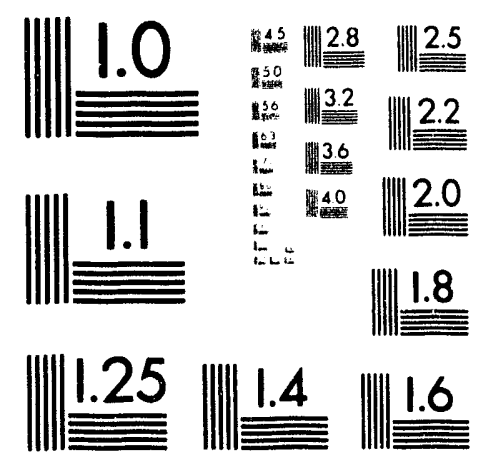



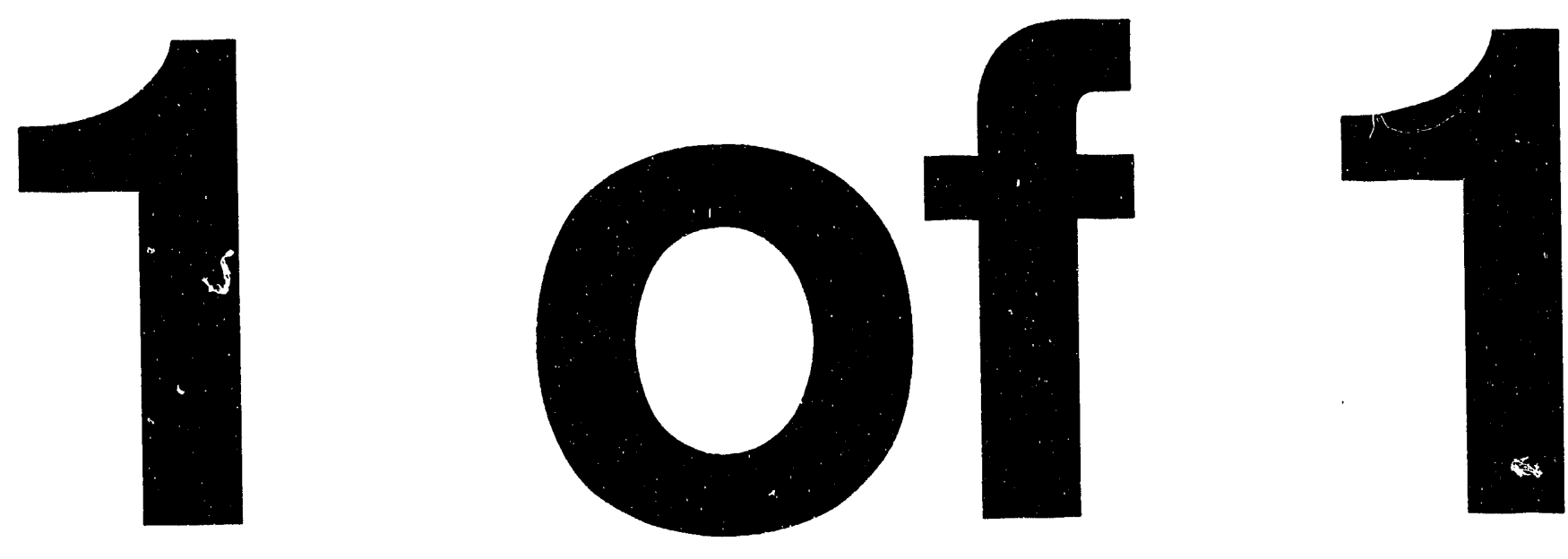


\title{
Geochemical Study of Evaporite and Clay Mineral-Oxyhydroxide Samples from the Waste Isolation Pilot Plant Site
}

\author{
Douglas G. Brookins \\ Department of Geology \\ University of New Mexico \\ Albuquerque, NM 87131
}

\begin{abstract}
Samples of clay minerals, insoluble oxyhydroxides, and their host evaporites from the WIPP site have been studied for their major and minor elements abundances, x-ray diffraction characteristics, $\mathrm{K}-\mathrm{Ar}$ ages, and $\mathrm{Rb}-\mathrm{Sr}$ ages. This study was undertaken to determine their overall geochemical characteristics and to investigate possible interactions between evaporites and insoluble constituents.

The evaporite host material is water-soluble, having $\mathrm{Cl} / \mathrm{Br}$ ratios typical of marine evaporites, although the $\mathrm{Br}$ content is low. Insoluble material (usually a mixture of clay minerals and oxyhydroxide phases) yields very high $\mathrm{Cl} / \mathrm{Br}$ ratios, possibly because of $\mathrm{Cl}$ from admixed halide minerals. This same material yields $\mathrm{K} / \mathrm{Rb}$ and $\mathrm{Th} / \mathrm{U}$ ratios in the normal range for shales; suggesting little, if any, effect of evaporite-induced remobilization of $\mathrm{U}, \mathrm{K}$, or $\mathrm{Rb}$ in the insoluble material. The rare-earth element (REE) data also show normal REE/chondrite (REE/CHON) distribution patterns, supporting the $\mathrm{K} / \mathrm{Rb}$ and $\mathrm{Th} / \mathrm{U}$ data.

Clay minerals yield $\mathrm{K}$-Ar dates in the range 365 to $390 \mathrm{Ma}$ and a $\mathrm{Rb}-\mathrm{Sr}$ isochron age of $428 \pm 7$ Ma. These ages are well in excess of the 2:0- to 230-Ma formational age of the evaporites, and confirm the detrital origin of the clays. The ages also show that any evaporite or clay mineral reactions that might have occurred at or near the time of sedimentation and diagenesis were not sufficient to reset the $\mathrm{K}-\mathrm{Ar}$ and $\mathrm{Rb} \cdot \mathrm{Sr}$ systematics of the clay minerals. Further, $\mathrm{X}$-ray data indicate a normal evaporitic assemblage of clay minerals and Fe-rich oxyhydroxide phases.

The clay minerals and other insoluble material appear to be resistant to the destructive effects of their entrapment in the evaporites, which suggests that these insoluble materials would be good getters for any radionuclides (hypothetically) released from the storage of radioactive wastes in the area.
\end{abstract}

*The work prescinted in this report was done by the late Dr. Brookins for Sandia National Laboratories under Contract No. 52-9984

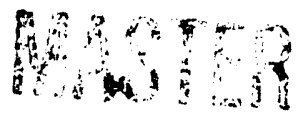




\section{Foreword}

This report is part of a series of contractor reports produced by the late Dr. Douglas Brookins and his associates (graduate students, research fellows, and colleagues), to provide detailed documentation of fundamental geochemical data collected to characterize the geological environment of the Waste Isolation Pilot Plant (WIPP). Previous works in this series have dealt with individual topics such as major and minor minerals (Register, 1981; Brookins and Lambert, 1987a; Brookins et al., 1990), potassium-argon and rubidium-strontium isochron dating (Brookins et al., 1980; Brookins, 1981; Brookins and Lambert, 1987b), strontium-isotope distributions determined by mass spectrometry (Brookins and Lambert, 1988), and rare-earth element distributions determined by neutron-activation analysis (Register and Brookins, 1980). Many of these studies have been pivotal in illuminating the nature, degree, and timing of the interactions between evaporite rocks and groundwaters in the Ochoan (Permian) evaporite section of the northern Delaware Basin, southeastern New Mexico.

Not only have most of these previous works been written on individual topics, but they have dealt with core recovered from exploratory boreholes as part of WIPP site characterization, which (except for limited investigations in nearby potash mines) was conducted entirely from the surface. Also, previous work has examined a variety of stratigraphic horizons, including those that bear witness to water/rock interactions in veining, paragenesis, and secondary mineralization. While consistent with the previous work, this mineralogical, elemental, and isotopic study represents a significant departure from that work. First, this work sampled only the horizon in the lower part of the Salado Formation (about 660 meters depth, just above marker bed 139) in the drifts excavated for WIPP. Second, this work uses many of the geochemical tools of the previous works, integrating the results into an internally consistent interpretation. Finally, this work illustrates that many of the previous results showed large variations because of regional heterogeneities that are less apparent on the scale of the WIPP facility itself. Nevertheless, the regional studies have proven justified to provide a more complete geologic history of the WIPP host-environment than can be obtained from only local sampling and investigation restricted to the WIPP itself.

Steven J. Lambert

Sandia National Laboratories

\section{Acknowledgments}

Ediiorial handling of early forms of this document was facilitated by E. Roberta Voelker. The manuscript was critically reviewed by Karen Robinson and Craig Novak. Final editing and production was done by Daniel P. Garber. 


\section{Contents}

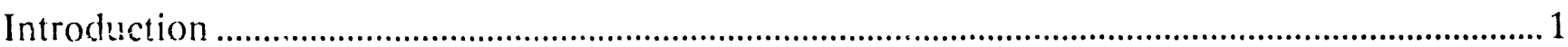

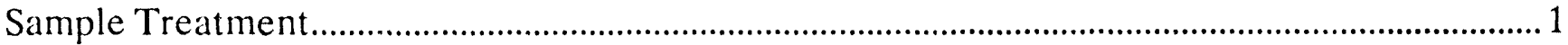

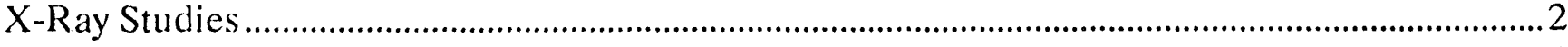

Elemental Geochemical Analysis .........................................................................................

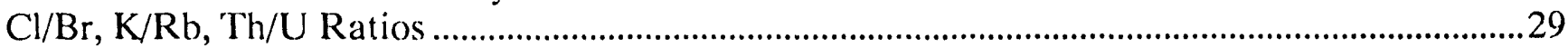

Studies of Rare-Earth Elements (REE).................................................................................

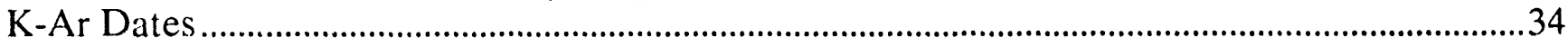

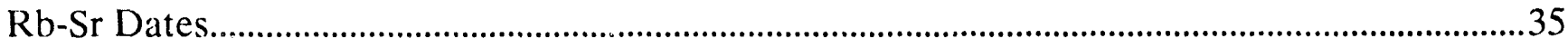

Conclusions: Implications of the Geochemistry of the Clay Minerals and Insoluble

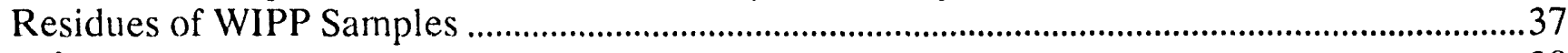

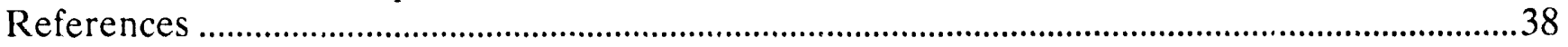

\section{Figures}

1 REE/CHON Normalized Distribution, Sample WP-1-C...................................................30

2 REE/CHON Normalized Distribution, Sample WP-2-C.....................................................30

3 REE/CHON Normalized Distribution, Sample WP-3-C..........................................................31

$4 \quad$ REE/CHON Normalized Distribution, Sample WP-4-C.....................................................31

5 REE/CHON Normalized Distribution, Sample WP-5-P .....................................................31

6 REE/CHON Normalized Distribution, Sample WP-7-C............................................................ 31

7 REE/CHON Normalized Distribution, Sample WP-8-C....................................................32

8 REE/CHON Normalized Distribution, Sample WP-9-C.........................................................32

$9 \quad \mathrm{REE} / \mathrm{CHON}$ Normalized Distribution, Sample FH-4-I............................................................32

10 REE/CHON Normalized Distribution, Sample FH-6B-I ................................................32

11 REE/CHON Normalized Distribution, Sample FH-27-C ........................................................33

12 REE/CHON Normalized Distribution, Sample FH-32-I..........................................................33

13 REE/CHON Normalized Distribution, Sample FH-42-C ......................................................33

14 REE/CHON Normalized Distribution, Sample FH-45-I........................................................33

$15 \mathrm{Rb}-\mathrm{Sr}$ Isochron for Clay Minerals and Oxyhydroxide Minerals .............................................36

\section{Tables}

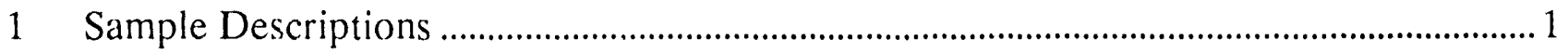

2 Mineralogy, from X-Ray Diffraction Data .........................................................................

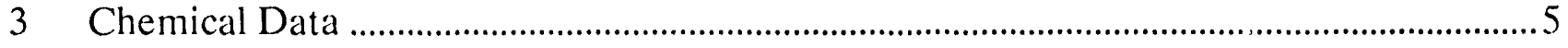

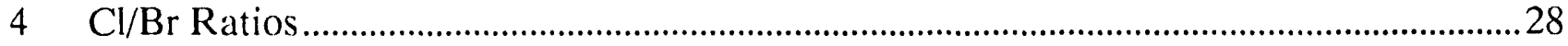

$5 \mathrm{Cl} / \mathrm{Br}, \mathrm{K} / \mathrm{Rb}$, and $\mathrm{Th} / \mathrm{U}$ Ratios for Insoluble Residues and Clay Minerals .........................29

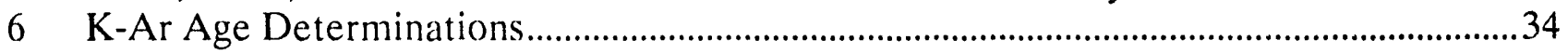

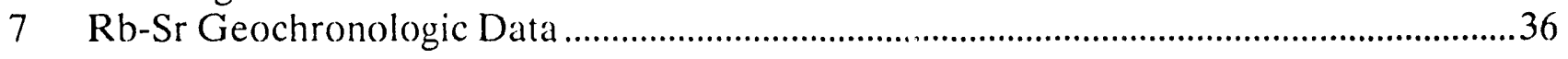




\section{Geochemical Study of Evaporite and Clay Mineral- Oxyhydroxide Samples from the Waste Isolation Pilot Plant (WIPP) Site}

\section{Introduction}

The purpose of this investigation is to obtain chemical, mineralogical, and geochronological data to assess the nature and history of the clay and other accessory minerals from the Waste Isolation Pilot Plant (WIPP) site area in southeastern New Mexico. For this purpose, samples of evaporites, most with clay minerals and other secondary or nonevaporitic minerals, were obtained from the underground WIPP-facilities.

\section{Sample Treatment}

The samples are briefly described in Table 1 . The series of samples designated "FH" were originally collected by IT Corporation during the WIPP Site and Preliminary Design Validation program, as the WIPP drifts were first excavated in the Salado Formation. The samples designated "WP" were collected on-site by the author with the assistance of S. J. Lambert.

Table 1. Sample Descriptions.

Sample

\section{WP-1}

WP-2

WP-3

WP-4

WP-5

WP-6

WP-7

WP-8

WP-9

WP-10

WP-11

(continued)

Location*

Brief Description

September 23, 1983.

N440-0E, Marker Unit 4, east rib $20 \mathrm{ft}$ south of WP-1, at break between Marker Units 4 and 5

N780, 40E, Marker Unit 4 N1100, OE, southeast corner, Marker Unit 4 at contact with Marker Unit 5

Room 1, $1250 \mathrm{~N}$, basal part of Marker Unit 3

Room 2, $1200 \mathrm{~N}$, on east wall

S3650, E140, between Marker Units 4 and 5

S2800, E140 same seam as WP-7

S2550, 140E

S400, 140E

From cage area
Very argillaceous halite

Gray clay seam

Massive brown argillaceous halite

Dark-gray clay seam

Polyhalite-bearing halite

Polyhalite-bearing halite

Dark-gray clay seam with minor halite

Brown-to-black clay seam

Polyhalite in break between brown and black clay seams

Reddish-brown clay seam

Polyhalite in halite 
Douglas G. Brookins

Table 1. (continued)

\begin{tabular}{|c|c|c|}
\hline Sample & Location* & Brief Description \\
\hline \multicolumn{3}{|c|}{ Samples from WIPP underground facilities collected during excavation } \\
\hline $\mathrm{FH} 4$ & OE drift, N53 ft, $4 \mathrm{ft}$ west of east rib & $\begin{array}{l}\text { Massive halite, some polyhalite } \\
\text { and clay }\end{array}$ \\
\hline $\mathrm{FH} 6 \mathrm{~A}$ & S90 drift, $145 \mathrm{ft}$ east, floor & Polyhalite from Unit 3 \\
\hline $\mathrm{FH} 6 \mathrm{~B}$ & S90 drift, $145 \mathrm{ft}$ east, floor & $\begin{array}{l}\text { Argillaceous halite from Units } 0 \\
\text { and } 1\end{array}$ \\
\hline $\mathrm{FH} 10$ & OE drift, S436 ft, east rib & $\begin{array}{l}\text { Clear halite, some polyhalite; } \\
\text { from Sandia corehole H1SE, 0-9 } \\
\text { and } 13-23 \text { inches depth }\end{array}$ \\
\hline $\mathrm{FH} 26$ & $\begin{array}{l}\text { E140 drift, } S 733 \mathrm{ft} \text {, back, } 8 \mathrm{ft} \text { east } \\
\text { of west rib }\end{array}$ & $\begin{array}{l}\text { Clear, coarsely crystalline halite, } \\
\text { trace clay, from Unit } 5\end{array}$ \\
\hline $\mathrm{FH} 27$ & $\begin{array}{l}\text { E140 drift, } \$ 1193 \mathrm{ft}, 11 \mathrm{ft} \text { from } \\
\text { west rib }\end{array}$ & $\begin{array}{l}\text { Halite with some polyhalite, from } \\
\text { Unit } 3(4 ?)\end{array}$ \\
\hline $\mathrm{FH} 32$ & $\begin{array}{l}\text { E140 drift, } S 1777 \mathrm{ft}, 10 \mathrm{ft} \text { from } \\
\text { east rib), } 4 \mathrm{ft} \text { below back }\end{array}$ & $\begin{array}{l}\text { Argillaceous, coarsely crystalline } \\
\text { halite with irregular masses of } \\
\text { gray clay, Unit } 4\end{array}$ \\
\hline $\mathrm{FH} 40$ & $\begin{array}{l}\text { Intersection of } O E \text { and } S 90 \text { drifts, } \\
\text { SE corner }\end{array}$ & $\begin{array}{l}\text { Argillaceous halite, medium to } \\
\text { coarsely crystalline, reddish- } \\
\text { brown with colorless halite, red- } \\
\text { dish-brown clay }\end{array}$ \\
\hline $\mathrm{FH} 42$ & Unknown & $\begin{array}{l}\text { Clear, coarsely crystalline halite; } \\
\text { minor reddish-brown clay }\end{array}$ \\
\hline $\mathrm{FH} 45$ & $\begin{array}{l}\text { E140 drift, } S 3080 \mathrm{ft} \text {, floor, } 3 \mathrm{ft} \\
\text { west of east rib }\end{array}$ & $\begin{array}{l}\text { Argillaceous halite; from vertical } \\
\text { downward corehole } \mathrm{DH}-224,0.45 \\
\mathrm{ft} \text { to } 1.0 \mathrm{ft} \text {, Unit } 0\end{array}$ \\
\hline
\end{tabular}

* relative to mine coordinates and informal marker units exposed in drift walls, as establishcd during the WIPP Site and Preliminary Design Validation.

The samples were split into two aliquots, whenever possible. The first aliquot was dissolved in water; the insoluble residue and the re-precipitated water-soluble fraction were analyzed separately. In some samples the clay minerals and other minerals (e.g., polyhalite) could be hand-separated from the evaporite minerals. The second aliquot, if available, was analyzed directly as a whole rock. Separate subaliquots of the above materials were selected for $\mathrm{x}$-ray study and for K-Ar and $\mathrm{Rb}-\mathrm{Sr}$ age-determination studies.

\section{X-Ray Studies}

Samples from both the FH and WP series were analyzed by $\mathrm{x}$-ray diffraction to determine the mineralogy, with emphasis on the insoluble residues (I) and/or clay-mineralrich separates (C) from the whole rocks. The data are presented in Table 2. The FH series samples, which were analyzed first, were not as well documented as the WP series. Hence, in Table $2 \mathrm{~A}$ no attempt is made to estimate the 
Table 2. Mineralogy, from X-Ray Diffraction Data.

\begin{tabular}{|c|c|c|c|c|c|c|c|c|}
\hline \multicolumn{9}{|c|}{ A. FH Series } \\
\hline Sample & HA & $\mathrm{PH}$ & GY & AN & SM & IL & $\mathrm{CH}$ & Q \\
\hline \multicolumn{9}{|c|}{ Insoluble residues and clays } \\
\hline $\mathrm{FH} 4-\mathrm{I}$ & - & - & $x$ & $x$ & - & - & - & $x$ \\
\hline FHGA-I & - & $x$ & $x$ & $x$ & - & - & - & - \\
\hline FH6B-C & - & - & - & - & $x$ & $x$ & $x$ & - \\
\hline $\mathrm{FH} 27-\mathrm{I}$ & - & - & $x$ & $x$ & - & - & - & - \\
\hline $\mathrm{FH} 27-\mathrm{C}$ & - & - & - & - & - & $x$ & $x$ & - \\
\hline $\mathrm{FH} 32-\mathrm{C}$ & - & - & - & - & - & $x$ & $x$ & $x$ \\
\hline $\mathrm{FH} 40-\mathrm{I}$ & $x$ & - & $x$ & $x$ & - & - & - & - \\
\hline $\mathrm{FH} 42-\mathrm{I}$ & $x$ & - & $x$ & $x$ & - & - & - & - \\
\hline $\mathrm{FH} 42-\mathrm{C}$ & - & - & - & - & $x$ & $x$ & $x$ & $x$ \\
\hline FH45-C & - & - & - & - & $x$ & $x$ & $x$ & $x$ \\
\hline \multicolumn{6}{|c|}{$\begin{array}{ll}\mathrm{X}-\text { present in readily identifiable amounts } & \mathrm{SM} \text { - smectite } \\
\mathrm{HA}-\text { halite } & \text { IL-illite } \\
\mathrm{PH} \text { - polyhalite } & \mathrm{CH} \text { - chlorite } \\
\text { GY - gypsum } & \mathrm{Q} \text {-quartz } \\
\text { AN - anhydrite } & \end{array}$} & & & \\
\hline
\end{tabular}

\section{B. WP Series}

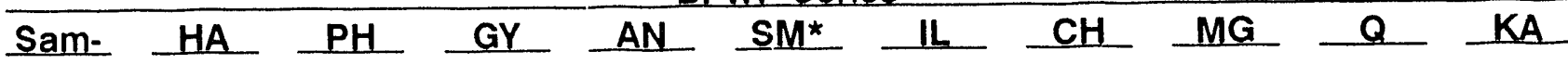
ple

\section{Clay separates and insoluble residues}

$\begin{array}{lllllllllll}\text { WP-1C } & - & - & - & - & M & X & t & t & M & - \\ \text { WP-2C } & - & - & - & - & M & X & - & x & M & - \\ \text { WP-3C } & - & - & - & - & M & M & - & t & X & - \\ \text { WP-4C } & - & - & - & - & M & X & t & t & M & - \\ \text { WP-7C } & - & - & - & - & M & X & t & t & M & - \\ \text { WP-8C } & - & - & - & - & M & M & - & t & X & - \\ \text { WP-9C } & - & - & - & - & M & X & t & t & M & - \\ \text { WP-10l } & - & - & - & - & M & X & - & t & M & -\end{array}$

(continued) 
Douglas G. Brookins

Table 2 (continued).

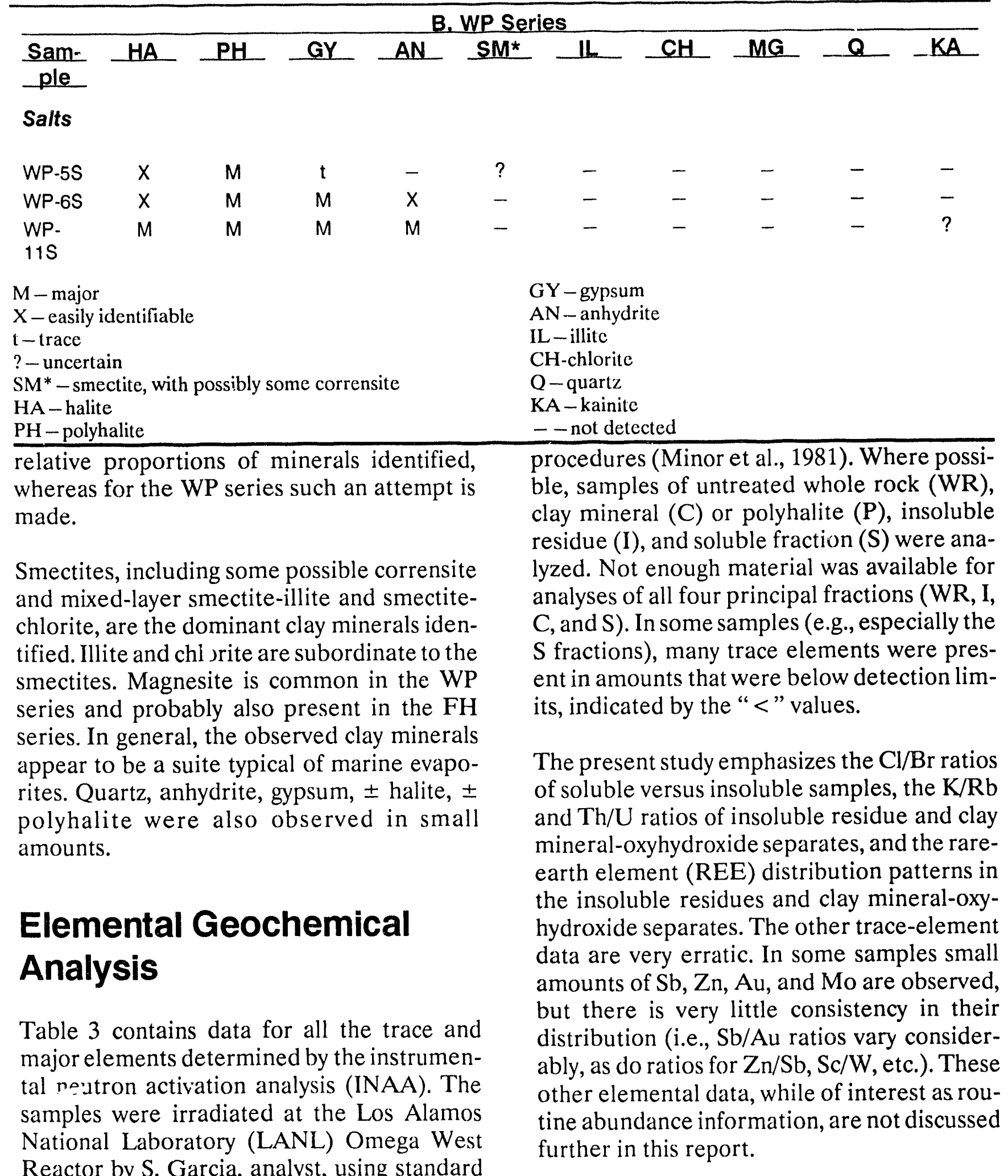


Table 3. Chemical Data. ${ }^{*}$

\begin{tabular}{|c|c|c|c|}
\hline \multicolumn{4}{|c|}{ A. Sample FH4 } \\
\hline Isotope & Soluble (S) & Insoluble (I) & Whole Rock (WR) \\
\hline $\mathrm{Na}-24$ & $262400 \pm 7600$ & $953 \pm 41$ & $323100 \pm 9000$ \\
\hline $\mathrm{Mg}-27$ & $<32000$ & $34900 \pm 1700$ & $<31000$ \\
\hline$A l-28$ & $<17000$ & $7950 \pm 520$ & $<16000$ \\
\hline $\mathrm{Cl}-38$ & $375000 \pm 21000$ & $960 \pm 63$ & $467000 \pm 26000$ \\
\hline K-42 & $<47000$ & $6400 \pm 720$ & $<45000$ \\
\hline $\mathrm{Ca}-49$ & $<17000$ & $177400 \pm 8000$ & $<18000$ \\
\hline Ti-51 & $<6400$ & $616 \pm 97$ & $<6300$ \\
\hline V-52 & $<110$ & $18.5 \pm 1.9$ & $<100$ \\
\hline$M n-56$ & $<16$ & $52.2 \pm 2.0$ & $<15$ \\
\hline Cu-66 & $<4100$ & $<110$ & $<3900$ \\
\hline Sr-87 & $<3000$ & $9840 \pm 740$ & $<3000$ \\
\hline $\ln -116$ & $<2.2$ & $<0.075$ & $<2.2$ \\
\hline $1-128$ & $<190$ & $<8.3$ & $<190$ \\
\hline Ba-139 & $<1500$ & $126 \pm 23$ & $<1400$ \\
\hline Dy-165 & $<14.0$ & $1.07 \pm 0.14$ & $<10.1$ \\
\hline U-235 & $<0.016$ & $0.483 \pm 0.014$ & $<0.0030$ \\
\hline Ga-72 & $<130$ & $<3.2$ & $<119$ \\
\hline As-76 & $<10.4$ & $0.620 \pm 0.082$ & $<9.2$ \\
\hline $\mathrm{Br}-82$ & $54.4 \pm 5.6$ & $0.66 \pm 0.11$ & $45.4 \pm 5.2$ \\
\hline Mo-99 & $<82$ & $<2.0$ & $<58$ \\
\hline La-140 & $<4.7$ & $19.45 \pm 0.70$ & $<4.2$ \\
\hline$S m-153$ & $<0.39$ & $3.44 \pm 0.16$ & $<0.25$ \\
\hline Yb-175 & $<5.5$ & $0.478 \pm 0.046$ & $<4.8$ \\
\hline W-187 & $<16.7$ & $<0.43$ & $<14.1$ \\
\hline Au-198 & $<0.12$ & $0.0200 \pm 0.0017$ & $<0.10$ \\
\hline Sc-46 & $<0.0123$ & $1.758 \pm 0.069$ & $0.0 .441 \leq 0.0035$ \\
\hline Cr-51 & $<6.1$ & $12.46 \pm 0.95$ & $<0.71$ \\
\hline $\mathrm{Fe}-59$ & $<240$ & $15070 \pm 570$ & $297 \pm 35$ \\
\hline Co-60 & $<0.0035$ & $1.145 \pm 0.085$ & $<0.0027$ \\
\hline $\mathrm{Zn}-65$ & $<0.12$ & $113+34$ & $\leq 0.080$ \\
\hline
\end{tabular}

* Data were taken by neutron activation analysis at Los Alamos National Laboratory's Omega West Reactor Site by S. Garcia, analyst. Data are reported in parts per million ( $\mathrm{ppm}$ ) unless noted otherwise. The isotopes listed on the page are those used for counting after the irradiations. Standards were independently run at LANL. The errors (" $\pm ")$ given are for one standard deviation, based on counting statistics. Where possible, three samples of each unknown were analyzed: (1) an aliquot of the whole rock (WR), (2) the soluble fraction (S) of a separate aliquot of sample (i.e., taken to dryness after dissolution and reprecipitated by evaporation), and (.3) the insoluble (I) or clay mineral-rich (C) or polyhalitic (P) separate of the sample aliquot from which the dissolution experiment was carried out.

(continued) 
Douglas G. Brookins

Table $s$ (continued).

\begin{tabular}{|c|c|c|c|}
\hline \multicolumn{4}{|c|}{ A. Sampie FH4 } \\
\hline isotope & Soluble (S) & Insoluble (I) & Whole Rock (WR) \\
\hline Se-75 & $<1.05$ & $<0.98$ & $<0.54$ \\
\hline $\mathrm{Rb}-86$ & $<66$ & $<8.0$ & $<3.2$ \\
\hline Zr-95 & $<88$ & $<93$ & $<31$ \\
\hline $\mathrm{Ag}-110$ & $<0.49$ & $<1.02$ & $<0.35$ \\
\hline Sb-124 & $<0.37$ & $<0.29$ & $<0.131$ \\
\hline Cs-134 & $<0.121$ & $0.77 \pm 0.11$ & $<0.098$ \\
\hline $\mathrm{Ce}-141$ & $<3.2$ & $41.2 \pm 1.6$ & $<0.48$ \\
\hline $\mathrm{Nd}-147$ & $<830$ & $29.4 \pm 5.1$ & $<5.7$ \\
\hline Eu-152 & $<0.033$ & $0.484 \pm 0.044$ & $<0.027$ \\
\hline Tb-160 & $<0.0037$ & $0.225 \pm 0.062$ & $<0.055$ \\
\hline Lu-177 & $<98$ & $0.052 \pm 0.011$ & $<0.025$ \\
\hline$H f-181$ & $<0.35$ & $0.694 \pm 0.053$ & $<0.075$ \\
\hline Ta-182 & $<0.28$ & $<0.28$ & $<0.190$ \\
\hline $\mathrm{Hg}-203$ & $<0.73$ & $<0.42$ & $<0.19$ \\
\hline Th-233 & $<0.95$ & $1.341+0.074$ & $\leq 0.105$ \\
\hline
\end{tabular}

\begin{tabular}{|c|c|}
\hline \multicolumn{2}{|c|}{ B. Sample FH6A } \\
\hline Isotope & Soluble (S) \\
\hline $\mathrm{Na}-24$ & $328400 \pm 9200$ \\
\hline$M g-27$ & $<32000$ \\
\hline Al-28 & $<17000$ \\
\hline $\mathrm{Cl}-38$ & $477000 \pm 26000$ \\
\hline$K-42$ & $<46000$ \\
\hline $\mathrm{Ca}-49$ & $<17000$ \\
\hline Ti-51 & $<6100$ \\
\hline V-52 & $<100$ \\
\hline$M n-56$ & $<15$ \\
\hline $\mathrm{Cu}-66$ & $<4000$ \\
\hline $\operatorname{Sr}-87$ & $<2800$ \\
\hline $\ln -116$ & $<2.2$ \\
\hline $1-128$ & $<180$ \\
\hline Ba-139 & $<1300$ \\
\hline Dy-165 & $<9.6$ \\
\hline U-235 & $0.2070 \pm 0.0058$ \\
\hline Ga-72 & $<160$ \\
\hline As-76 & $<12$ \\
\hline $\mathrm{Br}-82$ & $27.3 \pm 4.0$ \\
\hline Mo-99 & $<94$ \\
\hline$L a-140$ & $<5.4$ \\
\hline
\end{tabular}

(continued from previous column)

\begin{tabular}{|ll|}
\hline \multicolumn{2}{c|}{ B. Sample FH6A } \\
\cline { 1 - 1 } Ism-153 & \multicolumn{1}{c|}{ Soluble (S) } \\
Yb-175 & $<0.45$ \\
W-187 & $<6.3$ \\
Au-198 & $<20$ \\
Sc-46 & $<0.14$ \\
Cr-51 & $<0.0058$ \\
Fe-59 & $<0.76$ \\
Co-60 & $<64$ \\
Zn-65 & $0.160 \pm 0.045$ \\
Se-75 & $<3.6$ \\
Rb-86 & $<0.56$ \\
Zr-95 & $<3.3$ \\
Ag-110 & $<34$ \\
Sb-124 & $<0.35$ \\
Cs-134 & $<0.140$ \\
Ce-141 & $<0.104$ \\
Nd-147 & $<0.49$ \\
Eu-152 & $<5.7$ \\
Tb-160 & $<0.027$ \\
Lu-177 & $<0.057$ \\
S & $<0.028$ \\
\hline
\end{tabular}

(continued next column)

(continued) 
Table 3 (continued).

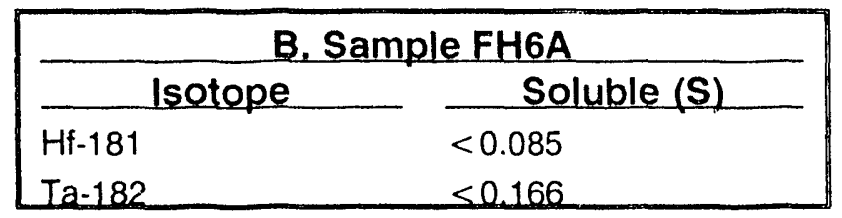

(continued next column) (continued from previous column)

\begin{tabular}{|c|c|}
\hline \multicolumn{2}{|c|}{ B. Sample FH6A } \\
\hline Isotope & Soluble (S) \\
\hline $\mathrm{Hg}-203$ & $<0.21$ \\
\hline Ih-233 & $<0116$ \\
\hline
\end{tabular}

\begin{tabular}{|c|c|c|c|}
\hline \multicolumn{4}{|c|}{ C. Sample FH6B } \\
\hline Isotope & Soluble (S) & Insoluble (I) & Whole Rock (WR) \\
\hline $\mathrm{Na}-24$ & $324400 \pm 9100$ & $1017 \pm 42$ & $298100 \pm 8600$ \\
\hline $\mathrm{Mg}-27$ & $<35000$ & $164900 \pm 6900$ & $<36000$ \\
\hline Al-28 & $<19000$ & $37600 \pm 1400$ & $<20000$ \\
\hline $\mathrm{Cl}-38$ & $474000 \pm 26000$ & $604 \pm 43$ & $440000 \pm 24000$ \\
\hline$K-42$ & $<52000$ & $6500 \pm 1200$ & $<54000$ \\
\hline $\mathrm{Ca}-49$ & $<20000$ & $4550 \pm 740$ & $<20000$ \\
\hline Ti-51 & $<6700$ & $2670 \pm 170$ & $<7300$ \\
\hline$V-52$ & $<120$ & $53.0 \pm 3.3$ & $<120$ \\
\hline$M n-56$ & $<16$ & $239.2 \pm 8.1$ & $<18$ \\
\hline $\mathrm{Cu}-66$ & $<4400$ & $<120$ & $<4600$ \\
\hline Sr-87 & $<3100$ & $<200$ & $<3400$ \\
\hline $\ln -116$ & $<2.4$ & $<0.082$ & $<2.5$ \\
\hline $1-128$ & $<200$ & $<13$ & $<220$ \\
\hline $\mathrm{Ba}-139$ & $<1400$ & $215 \pm 31$ & $<1700$ \\
\hline Dy-165 & $<10.2$ & $4.00 \pm 0.34$ & $<16$ \\
\hline U-235 & $0.1100 \pm 0.0031$ & $1.993 \pm 0.056$ & $0.0620 \pm 0.0017$ \\
\hline Ga-72 & $<140$ & $7.5 \pm 1.0$ & $<120$ \\
\hline As-76 & $<11.0$ & $2.19 \pm 0.19$ & $<9.0$ \\
\hline $\mathrm{Br}-82$ & $117.3 \pm 10.0$ & $2.35 \pm 0.21$ & $61.1 \pm 6.4$ \\
\hline Mo-99 & $<84$ & $1.36 \pm 0.17$ & $<56$ \\
\hline La-140 & $<4.9$ & $21.04 \pm 0.74$ & $<4.0$ \\
\hline Sm-153 & $<0.40$ & $3.83 \pm 0.20$ & $<0.24$ \\
\hline Yb-175 & $<5.6$ & $2.295 \pm 0.096$ & $<4.6$ \\
\hline W-187 & $<16.9$ & $0.86 \pm 0.16$ & $<13.2$ \\
\hline Au-198 & $<0.12$ & $<0.0034$ & $<0.099$ \\
\hline Sc-46 & $<0.00023$ & $7.30 \pm 0.28$ & $0.187 \pm 0.011$ \\
\hline $\mathrm{Cr}-51$ & $<1.0$ & $50.8 \pm 2.5$ & $<3.7$ \\
\hline Fe-59 & $<87$ & $25090 \pm 930$ & $613 \pm 78$ \\
\hline Co-60 & $<0.0047$ & $4.98 \pm 0.23$ & $0.239 \pm 0.036$ \\
\hline $\mathrm{Zn}-65$ & $<4.4$ & $<5.4$ & $24.1 \pm 4.1$ \\
\hline Se-75 & $<0.79$ & $<2.7$ & $<0.55$ \\
\hline $\mathrm{Bb}-86$ & $\leq 4.5$ & $42.3 \pm 4.7$ & $\leq 42$ \\
\hline
\end{tabular}

(continued) 
Douglas G. Brookins

Table 3 (continued).

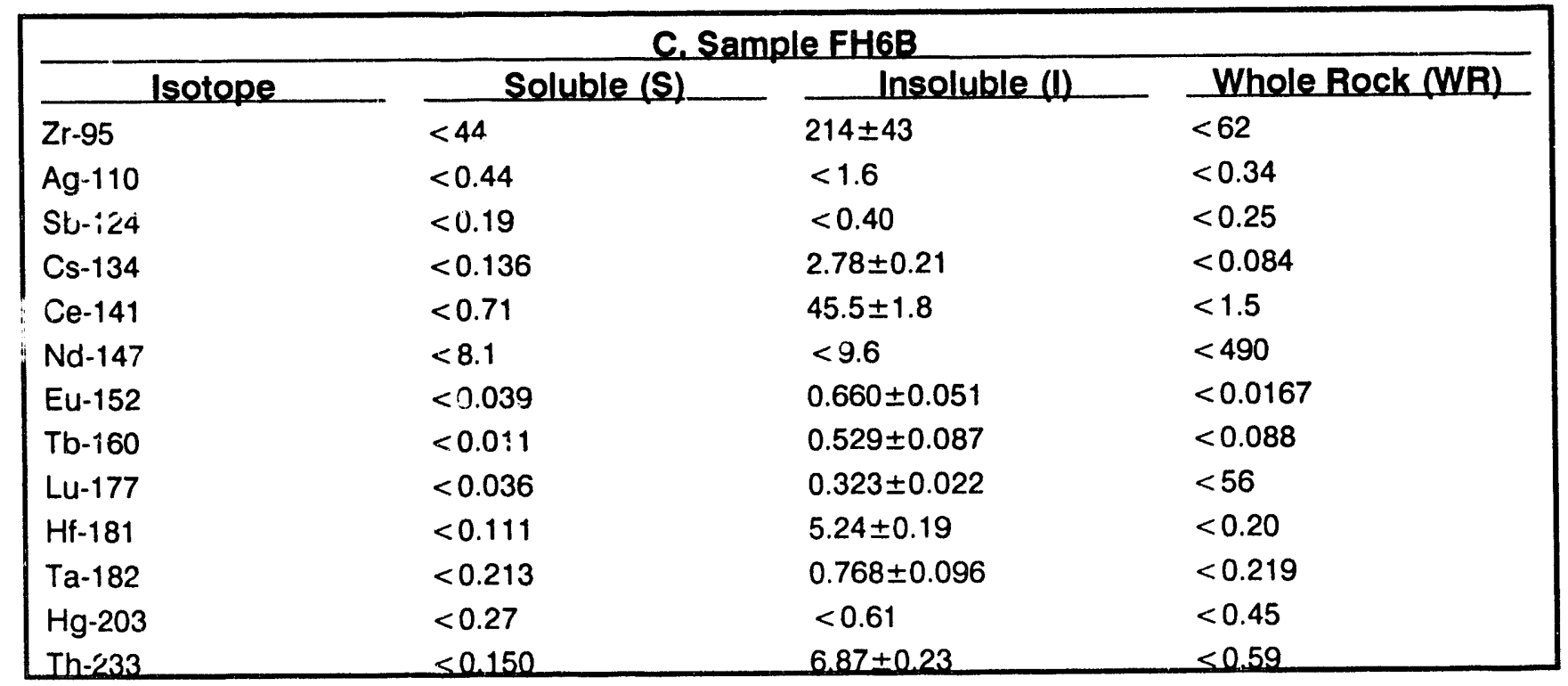

\begin{tabular}{|c|c|c|}
\hline \multicolumn{3}{|c|}{ D. Sample FH26 } \\
\hline Isotope & Soluble (S) & Whole Rock (WR) \\
\hline $\mathrm{Na}-24$ & $369000 \pm 10000$ & $335500 \pm 9400$ \\
\hline$M g-27$ & $<35000$ & $<31000$ \\
\hline Al-28 & $<19000$ & $<17000$ \\
\hline $\mathrm{Cl}-38$ & $527000 \pm 29000$ & $488000 \pm 27000$ \\
\hline$K-42$ & $<51000$ & $<45000$ \\
\hline $\mathrm{Ca}-49$ & $<21000$ & $<18000$ \\
\hline Ti-51 & $<7100$ & $<6000$ \\
\hline$V-52$ & $<120$ & $<100$ \\
\hline$M n-56$ & $<17$ & $<14$ \\
\hline $\mathrm{Cu}-66$ & $<4400$ & $<3900$ \\
\hline Sr 87 & $<3300$ & $<2800$ \\
\hline $\ln -116$ & $<2.5$ & $<2.1$ \\
\hline $1-128$ & $<210$ & $<180$ \\
\hline Ba-139 & $<1500$ & $<1200$ \\
\hline Dy-165 & $<11.4$ & $<9.3$ \\
\hline U-235 & $0.0700 \pm 0.0020$ & $0.1040 \pm 0.0029$ \\
\hline Ga-72 & $<190$ & $<121$ \\
\hline As-76 & $<14$ & $<8.8$ \\
\hline $\mathrm{Br}-82$ & $74.2 \pm 8.0$ & $43.4 \pm 4.6$ \\
\hline Mo-99 & $<106$ & $<54$ \\
\hline La-140 & $<6.0$ & $<3.9$ \\
\hline Sm-153 & $<0.51$ & $<0.24$ \\
\hline$Y b-175$ & $<6.9$ & $<4.4$ \\
\hline
\end{tabular}

(continued) 
Table 3 (continued).

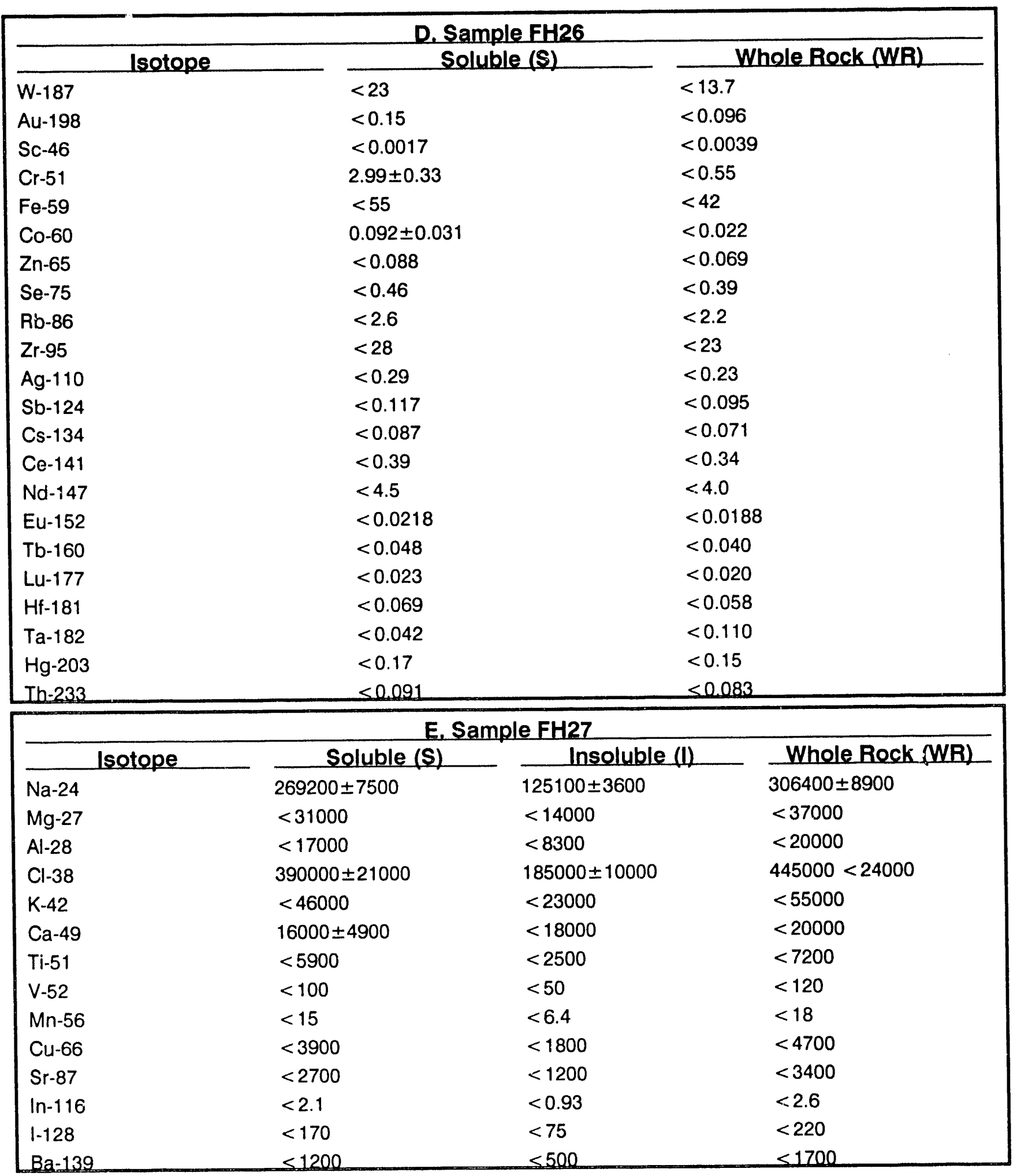

(continued) 
Dcuglas G. Brookins

Table 3 (continued).

\begin{tabular}{|c|c|c|c|}
\hline \multicolumn{4}{|c|}{ E. Sample FH27 } \\
\hline Isotope & Soluble (S) & Insoluble (I) & Whole Rock (WR) \\
\hline Dy-165 & $<9.0$ & $<3.6$ & $<16$ \\
\hline U-235 & $0.03500 \pm 0.00098$ & $3.409 \pm 0.095$ & $0.0370 \pm 0.0010$ \\
\hline Ga-72 & $<150$ & $7.6 \pm 1.5$ & $<10.7$ \\
\hline As-76 & $<11.2$ & $5.25 \pm 0.44$ & $<0.79$ \\
\hline $\mathrm{Br}-82$ & $36.4 \pm 5.5$ & $3.31 \pm 0.33$ & $3.45 \pm 0.37$ \\
\hline Mo-99 & $<85$ & $<4.1$ & $<4.8$ \\
\hline La-140 & $<4.9$ & $17.32 \pm 0.62$ & $<0.34$ \\
\hline Sm-153 & $<0.41$ & $3.38 \pm 0.16$ & $<0.021$ \\
\hline Yb-175 & $<5.6$ & $1.90 \pm 0.10$ & $<0.38$ \\
\hline W-187 & $<18.5$ & $<0.98$ & $<1.16$ \\
\hline$A u-198$ & $<012$ & $0.0078 \pm 0.0019$ & $<0.0085$ \\
\hline Sc-46 & $<0.00043$ & $9.29 \pm 0.35$ & $0.0058 \pm 0.0018$ \\
\hline $\mathrm{Cr}-51$ & $<7.6$ & $55.4 \pm 2.7$ & $<0.54$ \\
\hline $\mathrm{Fe}-59$ & $<310$ & $23170 \pm 860$ & $<45$ \\
\hline Co-60 & $<0.064$ & $9.38 \pm 0.40$ & $<0.016$ \\
\hline $\mathrm{Zn}-65$ & $<0.15$ & $<5.5$ & $<0.070$ \\
\hline Se-75 & $<1.31$ & $<2.4$ & $<0.38$ \\
\hline $\mathrm{Rb}-86$ & $<85$ & $39.9 \pm 4.5$ & $<2.3$ \\
\hline Zr-95 & $<105$ & $112 \pm 26$ & $<24$ \\
\hline $\mathrm{Ag}-110$ & $<0.58$ & $<1.8$ & $<0.24$ \\
\hline Sb-124 & $<0.46$ & $<0.51$ & $<0.099$ \\
\hline Cs-134 & $<0.144$ & $2.79 \pm 0.21$ & $<0.072$ \\
\hline Ce-141 & $<4.0$ & $32.1 \pm 1.3$ & $<0.32$ \\
\hline $\mathrm{Nd}-147$ & $<1030$ & $<9.3$ & $<3.1$ \\
\hline Eu-152 & $<0.041$ & $0.514 \pm 0.047$ & $<0.0177$ \\
\hline rb-160 & $<0.170$ & $0.48 \pm 0.12$ & $<0.040$ \\
\hline Lu-177 & $<129$ & $0.310 \pm 0.024$ & $<0.020$ \\
\hline $\mathrm{Hf}-181$ & $<0.42$ & $3.50 \pm 0.15$ & $<0.058$ \\
\hline Ta-182 & $<0.224$ & $0.70 \pm 0.11$ & $<0.109$ \\
\hline $\mathrm{Hg}-203$ & $<0.94$ & $<0.65$ & $<0.15$ \\
\hline Ih-233 & $<1.17$ & $7.48+0.25$ & $<0.085$ \\
\hline
\end{tabular}

\begin{tabular}{|c|c|c|c|}
\hline \multicolumn{4}{|c|}{ F. Sample FH32 } \\
\hline Isotope & Soluble (S) & Insoluble (I) & Whole Rock (WR) \\
\hline $\mathrm{Na}-24$ & $362000 \pm 10000$ & $4240 \pm 140$ & $316100 \pm 8900$ \\
\hline$M g-27$ & $<35000$ & $164500 \pm 7100$ & $<29000$ \\
\hline Al-28 & $<19000$ & $43900 \pm 1600$ & $<16000$ \\
\hline Cl-38 & $519000+29000$ & $3580+210$ & $463000+25000$ \\
\hline
\end{tabular}

(continued) 
Table 3 (continued).

\begin{tabular}{|c|c|c|c|}
\hline \multicolumn{4}{|c|}{ F. Sample FH32 } \\
\hline Isotope & Soluble (S) & Insoluble (I) & Whole Rock (WR) \\
\hline $\mathrm{K}-42$ & $<51000$ & $8100 \pm 1100$ & $<43000$ \\
\hline $\mathrm{Ca}-49$ & $<19000$ & $<440$ & $<17000$ \\
\hline Ti-51 & $<7100$ & $2180 \pm 190$ & $<5700$ \\
\hline$V-52$ & $<120$ & $55.7 \pm 3.7$ & $<98$ \\
\hline$M n-56$ & $<17$ & $341 \pm 12$ & $<14$ \\
\hline $\mathrm{Cu}-66$ & $<4400$ & $<180$ & $<3700$ \\
\hline Sr-87 & $<3300$ & $<250$ & $<2600$ \\
\hline $\ln -116$ & $<2.4$ & $<0.119$ & $<2.0$ \\
\hline $\mid-128$ & $<210$ & $<16$ & $<170$ \\
\hline Ba-139 & $<1500$ & $157 \pm 33$ & $<1200$ \\
\hline Dy-165 & $<11.4$ & $2.92 \pm 0.27$ & $<8.9$ \\
\hline U-235 & $0.0680 \pm 0.0019$ & $3.337 \pm 0.093$ & $0.0550 \pm 0.0015$ \\
\hline Ga-72 & $<140$ & $9.7 \pm 1.5$ & $<160$ \\
\hline As-76 & $<10.2$ & $5.59 \pm 0.44$ & $<11.1$ \\
\hline $\mathrm{Br}-82$ & $73.4 \pm 6.5$ & $2.64 \pm 0.27$ & $44.9 \pm 4.8$ \\
\hline Mo-99 & $<63$ & $2.73 \pm 0.29$ & $<82$ \\
\hline La-140 & $<4.3$ & $18.12 \pm 0.65$ & $<4.8$ \\
\hline Sm-153 & $<0.27$ & $2.78 \pm 0.13$ & $<0.40$ \\
\hline Yb-175 & $<5.1$ & $1.565 \pm 0.078$ & $<5.3$ \\
\hline$W-187$ & $<15.1$ & $1.70 \pm 0.31$ & $<18.3$ \\
\hline$A u-198$ & $<0.11$ & $0.0068 \pm 0.0020$ & $<0.12$ \\
\hline Sc-46 & $<0.0047$ & $8.32 \pm 0.32$ & $0.0379 \pm 0.0034$ \\
\hline Cr-51 & $<0.67$ & $51.0 \pm 2.5$ & $<0.76$ \\
\hline $\mathrm{Fe}-59$ & $<56$ & $21490 \pm 800$ & $138 \pm 33$ \\
\hline Co-60 & $<0.053$ & $10.02 \pm 0.42$ & $<0.00033$ \\
\hline$Z n-65$ & $<2.8$ & $36.5 \pm 5.9$ & $<0.089$ \\
\hline Se-75 & $<0.47$ & $<13$ & $<0.57$ \\
\hline $\mathrm{Rb}-86$ & $<2.8$ & $40.7 \pm 4.2$ & $<3.4$ \\
\hline Zr-95 & $<28$ & $<150$ & $<35$ \\
\hline $\mathrm{Ag}-110$ & $<0.29$ & $<1.7$ & $<0.35$ \\
\hline Sb-124 & $<0.114$ & $0.53 \pm 0.15$ & $<0.138$ \\
\hline Cs-134 & $0.053 \pm 0.026$ & $2.96 \pm 0.22$ & $<0.102$ \\
\hline Se-141 & $<0.40$ & $39.7 \pm 1.5$ & $<0.52$ \\
\hline $\mathrm{Nd}-147$ & $<5.0$ & $<8.8$ & $<6.1$ \\
\hline Eu-152 & $<0.0228$ & $0.510 \pm 0.045$ & $<0.028$ \\
\hline Tb-160 & $<0.048$ & $0.457 \pm 0.098$ & $<0.055$ \\
\hline $1 \mu-177$ & $<0.024$ & $0.243+0.020$ & $\leq 0.027$ \\
\hline
\end{tabular}

(continued) 
Douglas G. Brookins

Table 3 (continued).

\section{F. Sample FH32}

\begin{tabular}{|c|c|c|c|}
\hline Isotope & Soluble (S) & Insoluble (I) & Whole Rock (WR) \\
\hline $\mathrm{Hf}-181$ & $<0.071$ & $2.54 \pm 0.12$ & $<0.084$ \\
\hline Ta-182 & $<0.137$ & $0.607 \pm 0.088$ & $<0.212$ \\
\hline $\mathrm{Hg}-203$ & $<0.18$ & $<0.60$ & $<0.20$ \\
\hline Ih-233 & $\leq 0.102$ & $6.35+0.22$ & $\leq 0.112$ \\
\hline
\end{tabular}

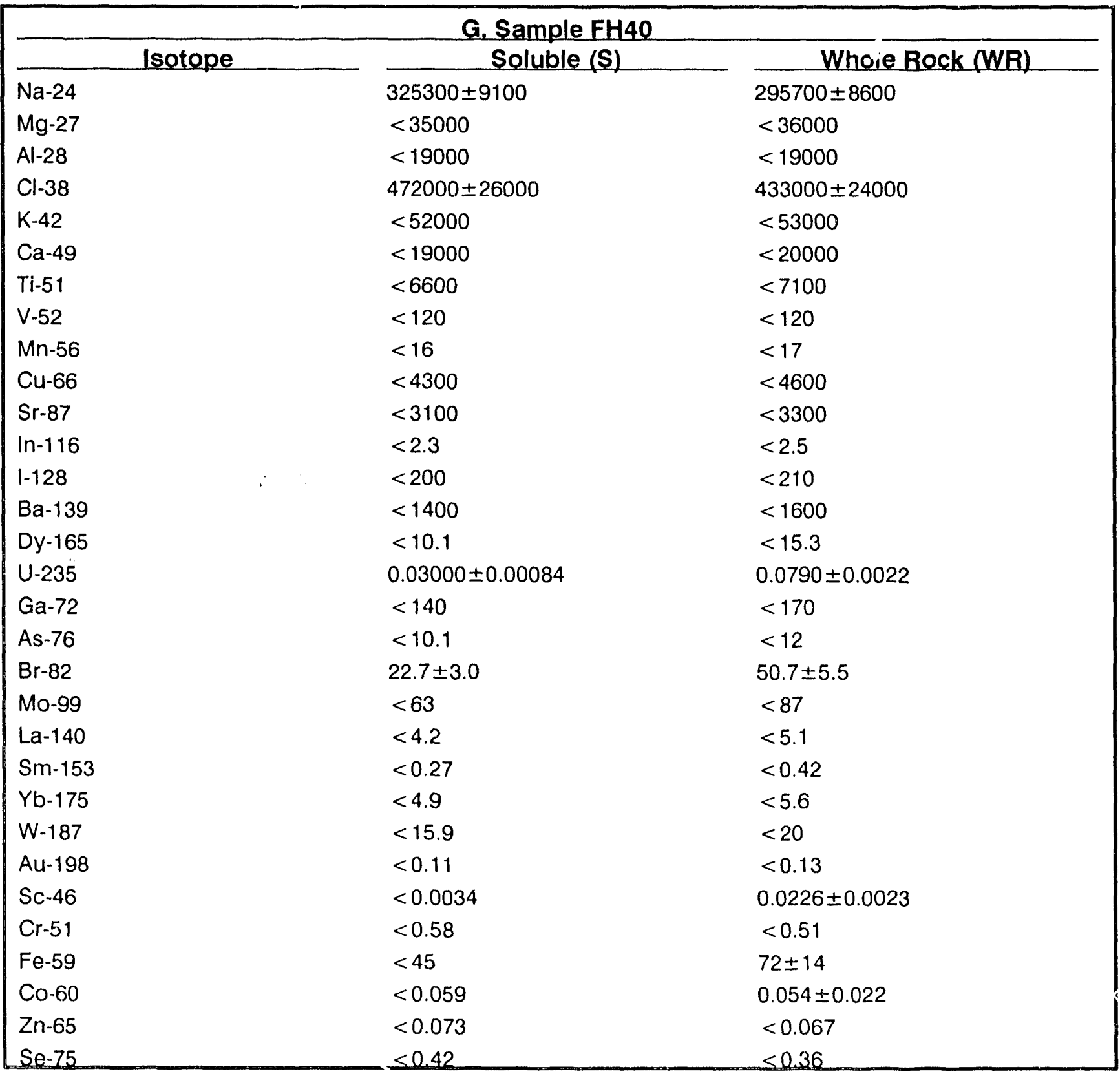

(continued) 
Table 3 (continued).

\begin{tabular}{|c|c|c|}
\hline \multicolumn{3}{|c|}{ G. Sample FH40 } \\
\hline Isotope & Soluble (S) & Whole Rock (WR) \\
\hline $\mathrm{Rb}-86$ & $<2.4$ & $<2.2$ \\
\hline Zr-95 & $<24$ & $<23$ \\
\hline $\mathrm{Ag}-110$ & $<0.26$ & $<0.23$ \\
\hline Sb-124 & $<0.103$ & $<0.093$ \\
\hline Cs-134 & $<0.075$ & $<0.069$ \\
\hline $\mathrm{Ce}-141$ & $<0.36$ & $<0.30$ \\
\hline $\mathrm{Nd}-147$ & $<4-5$ & $<4.0$ \\
\hline Eu-152 & $<0.0202$ & $<0.0177$ \\
\hline Tb-160 & $<0.038$ & $<0.035$ \\
\hline Lu-177 & $<0.021$ & $<0.018$ \\
\hline $\mathrm{Hf}-181$ & $<0.060$ & $<0.054$ \\
\hline Ta-182 & $<0.089$ & $<0.133$ \\
\hline $\mathrm{Hg}-203$ & $<0.16$ & $<0.13$ \\
\hline Th-233 & $\leq 0.087$ & $<0,079$ \\
\hline
\end{tabular}

\begin{tabular}{|c|c|c|c|}
\hline \multicolumn{4}{|c|}{ H. Sample FH42 } \\
\hline Isotope & Soluble (S) & Insoluble (I) & Whole Rock (WR) \\
\hline $\mathrm{Na}-24$ & $341700 \pm 9600$ & $8500 \pm 260$ & $323200 \pm 9000$ \\
\hline$M g-27$ & $<33000$ & $168100 \pm 7200$ & $<30000$ \\
\hline Al-28 & $<18000$ & $40100 \pm 1600$ & $<16000$ \\
\hline $\mathrm{Cl}-38$ & $496000 \pm 27000$ & $7470 \pm 420$ & $468000 \pm 26000$ \\
\hline $\mathrm{K}-42$ & $<49000$ & $4600 \pm 1100$ & $<44000$ \\
\hline $\mathrm{Ca}-49$ & $<19000$ & $<720$ & $<18000$ \\
\hline Ti-51 & $<6800$ & $2980 \pm 210$ & $<5900$ \\
\hline V-52 & $<110$ & $50.8 \pm 3.5$ & $<100$ \\
\hline$M n-56$ & $<16$ & $258.6 \pm 9.1$ & $<14$ \\
\hline Cu-66 & $<4200$ & $<250$ & $<3800$ \\
\hline Sr-87 & $<3100$ & $<270$ & $<2700$ \\
\hline $\ln -116$ & $<2.3$ & $<0.155$ & $<2.1$ \\
\hline $1-128$ & $<200$ & $<18$ & $<170$ \\
\hline Ba-139 & $<1500$ & $205 \pm 41$ & $<1200$ \\
\hline Dy-165 & $<10.8$ & $3.84 \pm 0.33$ & $<9.2$ \\
\hline U-235 & $0.0690 \pm 0.0019$ & $3.82 \pm 0.11$ & $0.0870 \pm 0.0024$ \\
\hline Ga-72 & $<160$ & $<7.3$ & $<140$ \\
\hline As-76 & $<12$ & $5.43 \pm 0.45$ & $<10.1$ \\
\hline $\mathrm{Br}-82$ & $138 \pm 11$ & $2.87 \pm 0.27$ & $54.3 \pm 5.2$ \\
\hline Mo-99 & $<72$ & $<3.7$ & $<61$ \\
\hline La-140 & $<4.8$ & $21.30 \pm 0.77$ & $<4.1$ \\
\hline $\mathrm{sm-153}$ & $\leq 0.31$ & $3.51+0.19$ & $\leq 0.27$ \\
\hline
\end{tabular}

(continued) 
Douglas G. Brookins

Table 3 (continued).

\begin{tabular}{|c|c|c|c|}
\hline \multicolumn{4}{|c|}{ H. Sample FH42 } \\
\hline Isotope & Soluble (S) & Insoluble (1) & Whole Rock (WR) \\
\hline$Y b-175$ & $<5.7$ & $2.113 \pm 0.099$ & $<4.8$ \\
\hline$W-187$ & $<17.6$ & $1.38 \pm 0.33$ & $<15.2$ \\
\hline Au-198 & $<0.13$ & $<0.0061$ & $<0.11$ \\
\hline Sc-46 & $<0.0072$ & $7.70 \pm 0.29$ & $0.1509 \pm 0.0074$ \\
\hline Cr-51 & $<0.99$ & $49.9 \pm 2.5$ & $1.55 \pm 0.26$ \\
\hline $\mathrm{Fe}-59$ & $<85$ & $14280 \pm 540$ & $299 \pm 25$ \\
\hline Co-60 & $<0.026$ & $8.76 \pm 0.39$ & $0.315 \pm 0.037$ \\
\hline$Z n-65$ & $<0.12$ & $<5.7$ & $<0.065$ \\
\hline Se-75 & $<0.78$ & $<3.1$ & $<0.38$ \\
\hline $\mathrm{Rb}-86$ & $<4.3$ & $38.6 \pm 4.6$ & $<2.5$ \\
\hline Zr-95 & $<44$ & $198 \pm 42$ & $<29$ \\
\hline $\mathrm{Ag}-110$ & $<0.41$ & $<1.7$ & $<0.30$ \\
\hline $\mathrm{Sb} 124$ & $<0.18$ & $0.84 \pm 025$ & $<0.110$ \\
\hline Cs-134 & $<0.133$ & $2.30 \pm 0.19$ & $0.097 \pm 0.028$ \\
\hline Ce-141 & $<0.71$ & $40.0 \pm 1.9$ & $1.08 \pm 0.12$ \\
\hline Nd-147 & $<7.9$ & $22.9 \pm$ & $<3.9$ \\
\hline Eu 152 & $<0.039$ & $0.742 \pm 0.058$ & $<0.0183$ \\
\hline Tb-160 & $<0.075$ & $0.613 \pm 0.089$ & $<0.046$ \\
\hline Lu-177 & $<0.037$ & $0.380 \pm 0.025$ & $<0.020$ \\
\hline $\mathrm{Hf}-181$ & $<0.113$ & $6.09 \pm 0.22$ & $0.144 \pm 0.023$ \\
\hline Ta-182 & $<0.201$ & $0.631 \pm 0.088$ & $<0.146$ \\
\hline $\mathrm{Hg}-203$ & $<0.28$ & $<0.64$ & $<0.15$ \\
\hline Ih-233 & & $738+0.25$ & $0.152+0.020$ \\
\hline \multicolumn{4}{|c|}{ 1. Sample FH45 } \\
\hline Isotope & Soluble (S) & Insoluble (I) & Whole Rock (WR) \\
\hline $\mathrm{Na}-24$ & $348700 \pm 9800$ & $10020 \pm 300$ & $326800 \pm 9500$ \\
\hline$M g-27$ & $<38000$ & $155900 \pm 6700$ & $<39000$ \\
\hline Al-28 & $<21000$ & $40100 \pm 1600$ & $<21000$ \\
\hline $\mathrm{Cl}-38$ & $511000 \pm 28000$ & $9830 \pm 550$ & $475000 \pm 26000$ \\
\hline $\mathrm{K}-42$ & $<57000$ & $7900 \pm 1300$ & $<57000$ \\
\hline $\mathrm{Ca}-49$ & $<22000$ & $<770$ & $<22000$ \\
\hline Ti-51 & $<7300$ & $2760 \pm 210$ & $<7500$ \\
\hline V-52 & $<130$ & $48.7 \pm 3.7$ & $<130$ \\
\hline$M n-56$ & $<18$ & $263.6 \pm 9.2$ & $<19$ \\
\hline Cu-66 & $<4900$ & $<270$ & $<4900$ \\
\hline Sr-87 & $<3400$ & $<270$ & $<3600$ \\
\hline $\ln -116$ & $\leq 26$ & $<0.164$ & $\leq 2.7$ \\
\hline
\end{tabular}

(continued) 
Table 3 (continued).

\begin{tabular}{|c|c|c|c|}
\hline \multicolumn{4}{|c|}{ 1. Sample FH45 } \\
\hline Isotope & Soluble (S) & Insoluble (I) & Whole Rock (WR) \\
\hline $1-128$ & $<220$ & $<18$ & $<230$ \\
\hline $\mathrm{Ba}-139$ & $<1500$ & $219 \pm 40$ & $<1800$ \\
\hline Dy-165 & $<11.2$ & $3.88 \pm 0.34$ & $<17$ \\
\hline U-235 & $0.0800 \pm 0.0022$ & $1.799 \pm 0.050$ & $0.0960 \pm 0.0027$ \\
\hline Ga-72 & $<170$ & $<8.2$ & $<116$ \\
\hline As-76 & $<11.5$ & $2.31 \pm 0.25$ & $<8.1$ \\
\hline $\mathrm{Br}-82$ & $118 \pm 10$ & $4.65 \pm 0.41$ & $46.0 \pm 4.3$ \\
\hline Mo-99 & $<66$ & $<4.7$ & $<47$ \\
\hline La-140 & $<4.8$ & $19.77 \pm 0.71$ & $<3.3$ \\
\hline Sm-153 & $<0.29$ & $3.20 \pm 0.17$ & $<0.21$ \\
\hline Yb-175 & $<5.3$ & $2.21 \pm 0.11$ & $<3.7$ \\
\hline$W-187$ & $<17.0$ & $<1.13$ & $<12.6$ \\
\hline Au-198 & $<0.12$ & $0.0163 \pm 0.0020$ & $<0.083$ \\
\hline Sc-46 & $0.0166 \pm 0.0030$ & $7.63 \pm 0.29$ & $0.0897 \pm 0.0065$ \\
\hline Cr-51 & $<1.00$ & $48.8 \pm 2.4$ & $<4.0$ \\
\hline $\mathrm{Fe}-59$ & $<86$ & $27200 \pm 1000$ & $940 \pm 150$ \\
\hline Co-60 & $<0.0046$ & $6.20 \pm 0.28$ & $0.240 \pm 0.045$ \\
\hline $\mathrm{Zn}-65$ & $<0.12$ & $<5.3$ & $<0.085$ \\
\hline Se-75 & $<0.77$ & $<2.7$ & $<0.65$ \\
\hline $\mathrm{Rb}-86$ & $<4.5$ & $43.2 \pm 4.8$ & $<46$ \\
\hline Zr-95 & $<43$ & $<150$ & $<61$ \\
\hline $\mathrm{Ag}-110$ & $<0.48$ & $<1.7$ & $<0.34$ \\
\hline Sb-124 & $<0.18$ & $0.97 \pm 0.25$ & $<0.25$ \\
\hline Cs-134 & $<0.131$ & $3.03 \pm 0.22$ & $<0.081$ \\
\hline $\mathrm{Ce}-141$ & $<0.70$ & $37.3 \pm 1.5$ & $<1.9$ \\
\hline $\mathrm{Nd}-147$ & $<7.9$ & $<9.8$ & $<580$ \\
\hline Eu-152 & $<0.039$ & $0.636 \pm 0.052$ & $<0.0195$ \\
\hline Tb-160 & $<0.074$ & $0.71 \pm 0.12$ & $<0.092$ \\
\hline Lu-177 & $<0.037$ & $0.349 \pm 0.025$ & $<71$ \\
\hline $\mathrm{Hf}-181$ & $<0.108$ & $4.34 \pm 0.16$ & $<0.23$ \\
\hline Ta-182 & $<0.220$ & $0.68 \pm 0.10$ & $<0.222$ \\
\hline $\mathrm{Hg}-203$ & $<0.27$ & $<0.63$ & $<0.51$ \\
\hline Th-233 & $50 \div 48$ & $6.51+0.23$ & $<0.63$ \\
\hline
\end{tabular}


Douglas G. Brookins

Table 3 (continued).

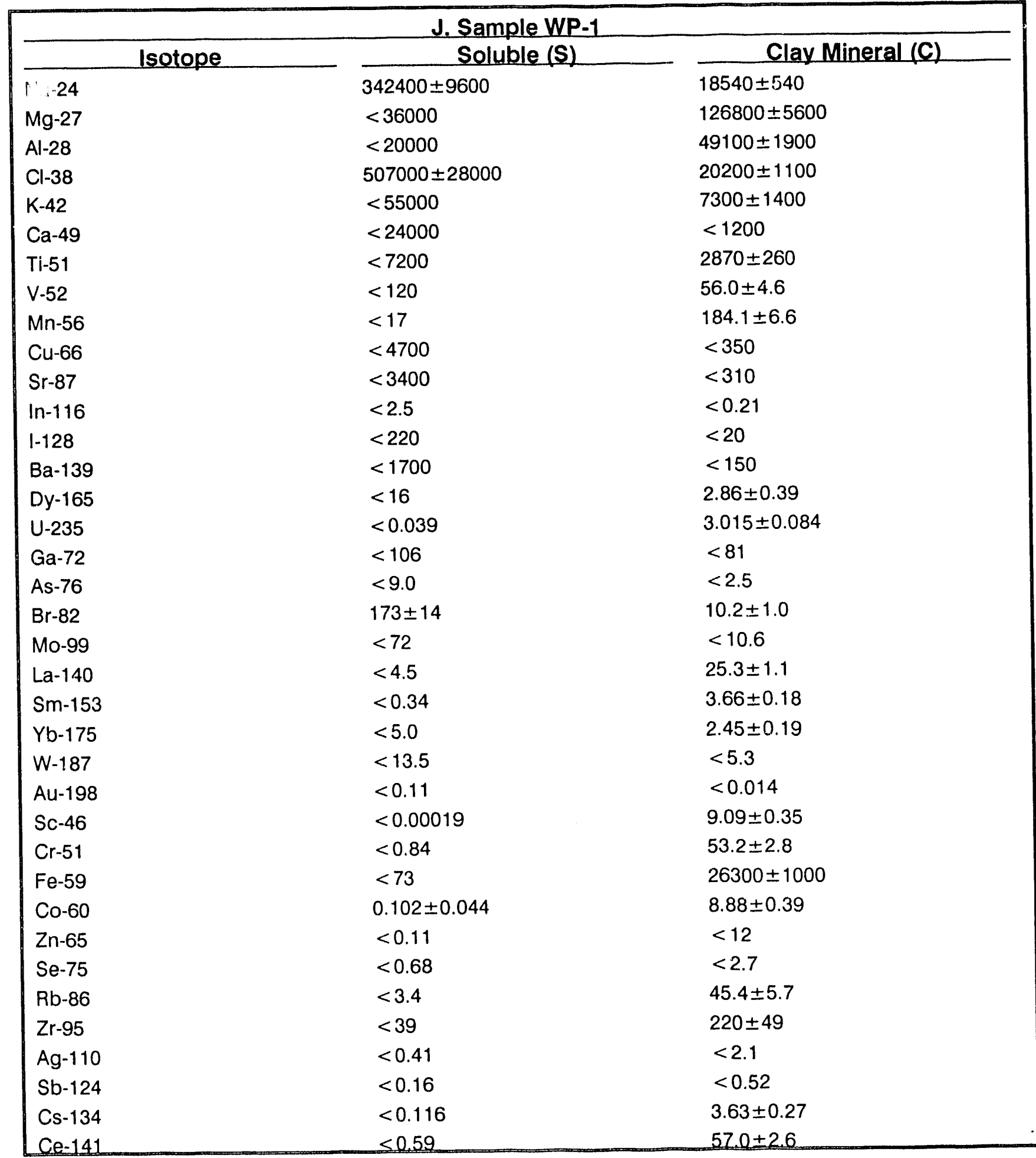

(continued) 
Table 3 (continued).

\begin{tabular}{|c|c|c|}
\hline & J. Sample WP-1 & \\
\hline Isotope & Soluble (S) & Clay Mineral (C) \\
\hline $\mathrm{Nd}-147$ & $<5.9$ & $<15$ \\
\hline Eu-152 & $0.152 \pm 0.033$ & $0.879 \pm C 069$ \\
\hline Tb-160 & $<0.066$ & $0.71 \pm 0.11$ \\
\hline Lu-177 & $<0.023$ & $0.375 \pm 0.028$ \\
\hline $\mathrm{Hf}-181$ & $<0.095$ & $5.38 \pm 0.22$ \\
\hline Ta-182 & $<0.108$ & $0.81 \pm 0.11$ \\
\hline $\mathrm{Hg}-203$ & $<0.23$ & $<0.79$ \\
\hline Th-233 & $<0.124$ & $8.16 \pm 0.29$ \\
\hline
\end{tabular}

\begin{tabular}{|c|c|c|}
\hline & K. Sample WP-2 & \\
\hline Isotope & Soluble (S) & Clay Mineral (C) \\
\hline $\mathrm{Na}-24$ & $351900 \pm 9900$ & $7400 \pm 220$ \\
\hline$M g-27$ & $<34000$ & $180900 \pm 7600$ \\
\hline Al-28 & $<19000$ & $37400 \pm 1500$ \\
\hline $\mathrm{Cl}-38$ & $514000 \pm 28000$ & $5900 \pm 340$ \\
\hline $\mathrm{K}-42$ & $<51000$ & $5100 \pm 1200$ \\
\hline $\mathrm{Ca}-49$ & $<24000$ & $<710$ \\
\hline Ti-51 & $<6300$ & $2360 \pm 200$ \\
\hline$V-52$ & $<110$ & $46.8 \pm 3.4$ \\
\hline$M n-56$ & $<16$ & $331 \pm 12$ \\
\hline Cu-66 & $<4200$ & $<230$ \\
\hline Sr-87 & $<2900$ & $<260$ \\
\hline $\ln -116$ & $<2.3$ & $<0.141$ \\
\hline $1-128$ & $<190$ & $<17$ \\
\hline Ba-139 & $<1300$ & $174 \pm 42$ \\
\hline Dy-165 & $<9.5$ & $3.44 \pm 0.31$ \\
\hline U-235 & $0.02700 \pm 0.00076$ & $2.383 \pm 0.067$ \\
\hline Ga-72 & $<240$ & $<64$ \\
\hline As-76 & $<6.9$ & $<2.0$ \\
\hline $\mathrm{Br}-82$ & $34.5 \pm 3.2$ & $3.63 \pm 0.59$ \\
\hline Mo-99 & $<22$ & $<8.3$ \\
\hline La-140 & $<2.1$ & $16.71 \pm 0.77$ \\
\hline Sm-153 & $<0.12$ & $2.77 \pm 0.14$ \\
\hline Yb-175 & $<1.6$ & $2.16 \pm 0.16$ \\
\hline$W-187$ & $<12.2$ & $<3.8$ \\
\hline Au-198 & $<0.040$ & $<0.011$ \\
\hline Sc-46 & $<0.000014$ & $7.91 \pm 0.30$ \\
\hline$c_{-}-51$ & $<0.55$ & $45.7 \pm 24$ \\
\hline
\end{tabular}

(continued) 
Douglas G. Brookins

Table 3 (continued).

\begin{tabular}{|c|c|c|}
\hline \multicolumn{3}{|c|}{ K. Sample WP-2 } \\
\hline Isotope & Soluble (S) & Clay Mineral (C) \\
\hline $\mathrm{Fe}-59$ & $<52$ & $23820 \pm 910$ \\
\hline Co-60 & $0.106 \pm 0.030$ & $7.77 \pm 0.35$ \\
\hline $\mathrm{Zn}-65$ & $16.9 \pm 2.9$ & $30.0 \pm 6.5$ \\
\hline Se-75 & $<0.41$ & $<2.8$ \\
\hline $\mathrm{Rb}-86$ & $<2.5$ & $46.6 \pm 5.4$ \\
\hline $\mathrm{Zr}-95$ & $<26$ & $<180$ \\
\hline $\mathrm{Ag}-110$ & $<0.26$ & $<2.0$ \\
\hline Sb-124 & $<0.104$ & $<0.58$ \\
\hline Cs-134 & $<0.080$ & $2.42 \pm 0.22$ \\
\hline $\mathrm{Ce}-141$ & $<0.33$ & $39.2 \pm 1.6$ \\
\hline $\mathrm{Nd}-147$ & $<3.9$ & $<14$ \\
\hline Eu-152 & $<0.0200$ & $0.661 \pm 0.060$ \\
\hline Tb-160 & $<0.043$ & $0.528 \pm 0.087$ \\
\hline Lu-177 & $<0.017$ & $0.349 \pm 0.028$ \\
\hline $\mathrm{Hf}-181$ & $<0.063$ & $3.56 \pm 0.16$ \\
\hline Ta-182 & $<0.065$ & $0.64 \pm 0.12$ \\
\hline $\mathrm{Hg}-203$ & $<0.15$ & $<0.72$ \\
\hline $\operatorname{Ih}-233$ & & $6.56+0.24$ \\
\hline \multicolumn{3}{|c|}{ L. Sample WP-3 } \\
\hline Isotope & Soluble & Clay Mineral \\
\hline $\mathrm{Na}-24$ & $285100 \pm 8000$ & $15040 \pm 440$ \\
\hline $\mathrm{Mg}-27$ & $<31000$ & $137000 \pm 5900$ \\
\hline $\mathrm{Al}-28$ & $<17000$ & $52600 \pm 1900$ \\
\hline $\mathrm{Cl}-38$ & $444000 \pm 24000$ & $14390 \pm 810$ \\
\hline$K-42$ & $<47000$ & $9700 \pm 2000$ \\
\hline Ca-49 & $<23000$ & $<820$ \\
\hline $\mathrm{Ti}-51$ & $<6000$ & $3110 \pm 230$ \\
\hline$V-52$ & $<110$ & $64.3 \pm 4.6$ \\
\hline$M n-56$ & $<14$ & $189.7 \pm 6.8$ \\
\hline Cu-66 & $<3900$ & $<260$ \\
\hline Sr-87 & $<2800$ & $<240$ \\
\hline $\ln -116$ & $<2.1$ & $<0.151$ \\
\hline $\mathrm{I}-128$ & $<180$ & $<15$ \\
\hline Ba-139 & $<1400$ & $294 \pm 48$ \\
\hline Dy-165 & $<12.8$ & $4.00 \pm 0.35$ \\
\hline$U-235$ & $0.01000 \pm 0.00028$ & $2.688 \pm 0.075$ \\
\hline $\mathrm{Ga}-72$ & $<230$ & $\leq 78$ \\
\hline
\end{tabular}

(continued) 
Table 3 (continued).

\begin{tabular}{|c|c|c|}
\hline & L. Sample WP-3 & \\
\hline Isotope & Soluble & Clay Mineral \\
\hline As-76 & $<7.2$ & $<2.4$ \\
\hline $\mathrm{Br}-82$ & $591 \pm 44$ & $2.87 \pm 0.56$ \\
\hline Mo-99 & $<22$ & $<10.1$ \\
\hline La-140 & $<1.97$ & $25.4 \pm 1.1$ \\
\hline Sm-153 & $<0.11$ & $3.81 \pm 0.19$ \\
\hline$Y b-175$ & $<1.6$ & $2.33 \pm 0.17$ \\
\hline$W-187$ & $<11.5$ & $<5.3$ \\
\hline$A u-i 98$ & $<0.041$ & $<0.013$ \\
\hline Sc-46 & $<0.0014$ & $8.88 \pm 0.34$ \\
\hline $\mathrm{Cr}-51$ & $<0.54$ & $55.2 \pm 2.8$ \\
\hline $\mathrm{Fe}-59$ & $<45$ & $33900 \pm 1300$ \\
\hline Co-60 & $0.123 \pm 0.031$ & $7.13 \pm 0.33$ \\
\hline $2 n-65$ & $14.7 \pm 2.7$ & $<12$ \\
\hline Se-75 & $<0.40$ & $<3.1$ \\
\hline $\mathrm{Rb}-86$ & $6.4 \pm 1.3$ & $39.7 \pm 5.1$ \\
\hline Zr-95 & $<25$ & $<190$ \\
\hline $\mathrm{Ag}-110$ & $<0.26$ & $<2.1$ \\
\hline Sb-124 & $<0.099$ & $<0.50$ \\
\hline Cs-134 & $0.227 \pm 0.038$ & $3.30 \pm 0.28$ \\
\hline $\mathrm{Ce}-141$ & $<0.32$ & $56.8 \pm 2.2$ \\
\hline $\mathrm{Nd}-147$ & $<3.9$ & $26.0 \pm 6.8$ \\
\hline Eu-152 & $0.152 \pm 0.022$ & $0.952 \pm 0.072$ \\
\hline Tb-160 & $<0.041$ & $0.54 \pm 0.14$ \\
\hline Lu-177 & $<0.017$ & $0.402 \pm 0.031$ \\
\hline $\mathrm{Hf}-181$ & $<0.060$ & $5.11 \pm 0.20$ \\
\hline Ta-182 & $<0.0334$ & $0.70 \pm 0.10$ \\
\hline $\mathrm{Hg}-203$ & $<0.15$ & $<0.78$ \\
\hline Th-233 & $<0.081$ & $7.82 \pm 0.27$ \\
\hline \multicolumn{3}{|c|}{ M. Sample WP-4 } \\
\hline Isotope & Soluble (S) & Clay Mineral (C) \\
\hline $\mathrm{Na}-24$ & $321700 \pm 9000$ & $18310 \pm 530$ \\
\hline$M g-27$ & $<31000$ & $138900 \pm 6100$ \\
\hline Al-28 & $<17000$ & $57600 \pm 2100$ \\
\hline $\mathrm{Cl}-38$ & $471000 \pm 26000$ & $18700 \pm 1000$ \\
\hline $\mathrm{K}-42$ & $<47000$ & $9100 \pm 1500$ \\
\hline $\mathrm{Ca}-49$ & $<23000$ & $<1200$ \\
\hline$T i-51$ & $<5800$ & $3260+310$ \\
\hline
\end{tabular}

(continued) 
Douglas G. Brookins

Table 3 (c sntinued).

\begin{tabular}{|c|c|c|}
\hline & M. Sample V.P-4 & \\
\hline Isotope & Soluble (S) & Clay Mineral (C) \\
\hline$V-52$ & $<100$ & $66.3 \pm 4.6$ \\
\hline$M n-56$ & $<14$ & $237.7 \pm 8.3$ \\
\hline Cu-66 & $<3900$ & $<360$ \\
\hline Sr-87 & $<2700$ & $<320$ \\
\hline $\ln -116$ & $<2.1$ & $<0.21$ \\
\hline $1-128$ & $<170$ & $<21$ \\
\hline Ba-139 & $<1200$ & $<150$ \\
\hline Dy-165 & $<8.7$ & $3.33 \pm 0.47$ \\
\hline U-235 & $<0.024$ & $3.97 \pm 0.11$ \\
\hline Ga-72 & $<260$ & $<85$ \\
\hline As-76 & $<7.4$ & $5.80 \pm 0.84$ \\
\hline $\mathrm{Br}-82$ & $253 \pm 19$ & $2.88 \pm 0.66$ \\
\hline Mo-99 & $<23$ & $<10.5$ \\
\hline La-140 & $<2.1$ & $23.1 \pm 1.0$ \\
\hline Sm-153 & $<0.12$ & $3.51 \pm 0.17$ \\
\hline Yb-175 & $<1.6$ & $2.06 \pm 0.16$ \\
\hline W-187 & $<12.3$ & $<5.0$ \\
\hline Au-198 & $<0.042$ & $<0.014$ \\
\hline Sc-46 & $<0.0013$ & $10.10 \pm 0.38$ \\
\hline Cr-51 & $<0.62$ & $61.8 \pm 3.2$ \\
\hline Fe-59 & $<56$ & $25120 \pm 950$ \\
\hline Co-60 & $0.184 \pm 0.045$ & $10.15 \pm 0.45$ \\
\hline $\mathrm{Zn}-65$ & $16.8 \pm 3.2$ & $<13$ \\
\hline Se-75 & $<0.47$ & $<2.2$ \\
\hline $\mathrm{Rb}-86$ & $<2.6$ & $57.0 \pm 5.8$ \\
\hline Zr-95 & $<31$ & $<200$ \\
\hline $\mathrm{Ag}-110$ & $<0.30$ & $<2.2$ \\
\hline Sb-124 & $<0.122$ & $<0.57$ \\
\hline Cs-134 & $<0.092$ & $3.75 \pm 0.28$ \\
\hline $\mathrm{Ce}-141$ & $<0.38$ & $53.5 \pm 2.1$ \\
\hline $\mathrm{Nd}-147$ & $<4.4$ & $28.7 \pm 8.0$ \\
\hline Eu-152 & $<0.0226$ & $0.852 \pm 0.072$ \\
\hline Tb-160 & $<0.049$ & $0.56 \pm 0.10$ \\
\hline Lu-177 & $<0.019$ & $0.330 \pm 0.028$ \\
\hline$H f-181$ & $<0.071$ & $4.36 \pm 0.19$ \\
\hline Ta-182 & $<0.0384$ & $0.79 \pm 0.12$ \\
\hline $\mathrm{Ha}-203$ & 50.18 & $\leq 0.82$ \\
\hline
\end{tabular}

(continued) 
Table 3 (continued).

\begin{tabular}{|c|c|c|}
\hline \multicolumn{3}{|c|}{ M. Sample WP-4 } \\
\hline Isotope & Soluble (S) & Clay Mineral (C) \\
\hline Th-233 & $<0.094$ & $8.82+0.31$ \\
\hline
\end{tabular}

\begin{tabular}{|c|c|c|}
\hline \multicolumn{3}{|c|}{ N. Sample WP-5 } \\
\hline Isotope & Soluble (S) & Polyhalite (P) \\
\hline $\mathrm{Na}-24$ & $2881000 \pm 8100$ & $332 \pm 18$ \\
\hline$M g-27$ & $<29000$ & $38300 \pm 1800$ \\
\hline Al-28 & $<17000$ & $<270$ \\
\hline $\mathrm{Cl}-38$ & $422000 \pm 23000$ & $70 \pm 10$ \\
\hline$K-42$ & $<46000$ & $120200 \pm 5900$ \\
\hline $\mathrm{Ca}-49$ & $<26000$ & $128600 \pm 5500$ \\
\hline Ti-51 & $<5600$ & $<210$ \\
\hline V-52 & $<100$ & $<1.7$ \\
\hline$M n-56$ & $<14$ & $16.73 \pm 0.77$ \\
\hline Cu-66 & $<3700$ & $<78$ \\
\hline Sr-87 & $<2600$ & $6770 \pm 510$ \\
\hline $\ln -116$ & $<2.0$ & $<0.056$ \\
\hline $\mid-128$ & $<170$ & $<5.7$ \\
\hline $\mathrm{Ba}-139$ & $<1300$ & $<51$ \\
\hline Dy-165 & $<11.9$ & $<0.037$ \\
\hline U-235 & $<0.022$ & $0.02100 \pm 0.00059$ \\
\hline Ga-72 & $<230$ & $<20$ \\
\hline As-76 & $<6.4$ & $<0.64$ \\
\hline $\mathrm{Br}-82$ & $68.0 \pm 5.4$ & $<0.62$ \\
\hline Mo-99 & $<20$ & $<3.9$ \\
\hline La-140 & $<1.87$ & $5.26 \pm 0.31$ \\
\hline Sm-153 & $<0.11$ & $0.501 \pm 0.028$ \\
\hline Yb-175 & $<1.39$ & $<0.14$ \\
\hline W-187 & $<11.2$ & $<1.26$ \\
\hline $\mathrm{Au}-198$ & $<0.036$ & $<0.0038$ \\
\hline Sc-46 & $<0.00013$ & $0.0501 \pm 0.0035$ \\
\hline Cr-51 & $<0.52$ & $<0.86$ \\
\hline $\mathrm{Fe}-59$ & $<47$ & $385 \pm 33$ \\
\hline Co-60 & $0.132 \pm 0.036$ & $0.095 \pm 0.032$ \\
\hline $\mathrm{Zn}-65$ & $14.1 \pm 2.7$ & $<0.039$ \\
\hline Se-75 & $<0.38$ & $<0.62$ \\
\hline $\mathrm{Rb}-86$ & $<2.3$ & $<2.7$ \\
\hline Zr-95 & $\leq 24$ & $<30$ \\
\hline
\end{tabular}

(continued) 
Douglas G. Brookins

Table 3 (continued).

\begin{tabular}{|c|c|c|}
\hline \multicolumn{3}{|c|}{ N. Sample WP-5 } \\
\hline Isotope & Soluble (S) & Polyhalite (P) \\
\hline $\mathrm{Ag}-110$ & $<0.25$ & $<0.31$ \\
\hline Sb-124 & $<0.0 ! 37$ & $<0.117$ \\
\hline Cs-134 & $<0.0 \% 4$ & $<0.091$ \\
\hline $\mathrm{Ce}-141$ & $<0.31$ & $10.08 \pm 0.50$ \\
\hline Nd-147 & $<3.7$ & $<4.6$ \\
\hline Eu-152 & $<0.0187$ & $0.115 \pm 0.022$ \\
\hline Tb-160 & $<0.041$ & $<0.010$ \\
\hline Lu-177 & $<0.016$ & $<0.023$ \\
\hline$H f-181$ & $<0.054$ & $<0.079$ \\
\hline Ta-182 & $<0.053$ & $<0.170$ \\
\hline $\mathrm{Hg}-203$ & $<0.14$ & $<0.22$ \\
\hline Ih-233 & $<0.081$ & $<0.129$ \\
\hline
\end{tabular}

\begin{tabular}{|c|c|}
\hline \multicolumn{2}{|c|}{ 0. Sample WP-6 } \\
\hline Isotope & Soluble (S) \\
\hline $\mathrm{Na}-24$ & ND (no: determined) \\
\hline $\mathrm{Mg}-27$ & ND \\
\hline Al-28 & ND \\
\hline $\mathrm{Cl} \cdot 38$ & ND \\
\hline $\mathrm{K}-42$ & ND \\
\hline Ca-49 & ND \\
\hline Ti-51 & ND \\
\hline$V-52$ & ND \\
\hline$M r_{-}-56$ & ND \\
\hline $\mathrm{Cu}-66$ & ND \\
\hline Sr-87 & ND \\
\hline $\ln -116$ & ND \\
\hline $1-128$ & ND \\
\hline $\mathrm{Ba}-139$ & ND \\
\hline Dy-165 & ND \\
\hline U-235 & $0.001000 \pm 0.000028$ \\
\hline Ga-72 & $<250$ \\
\hline As-76 & $<6.9$ \\
\hline $\mathrm{Br}-82$ & $69.8 \pm 5.6$ \\
\hline Mo-99 & $<21$ \\
\hline La-140 & $<1.99$ \\
\hline Sm-153 & $<1) .11$ \\
\hline$Y b-175$ & $<1.5$ \\
\hline
\end{tabular}

(continued next column) (continued frum previn: column)

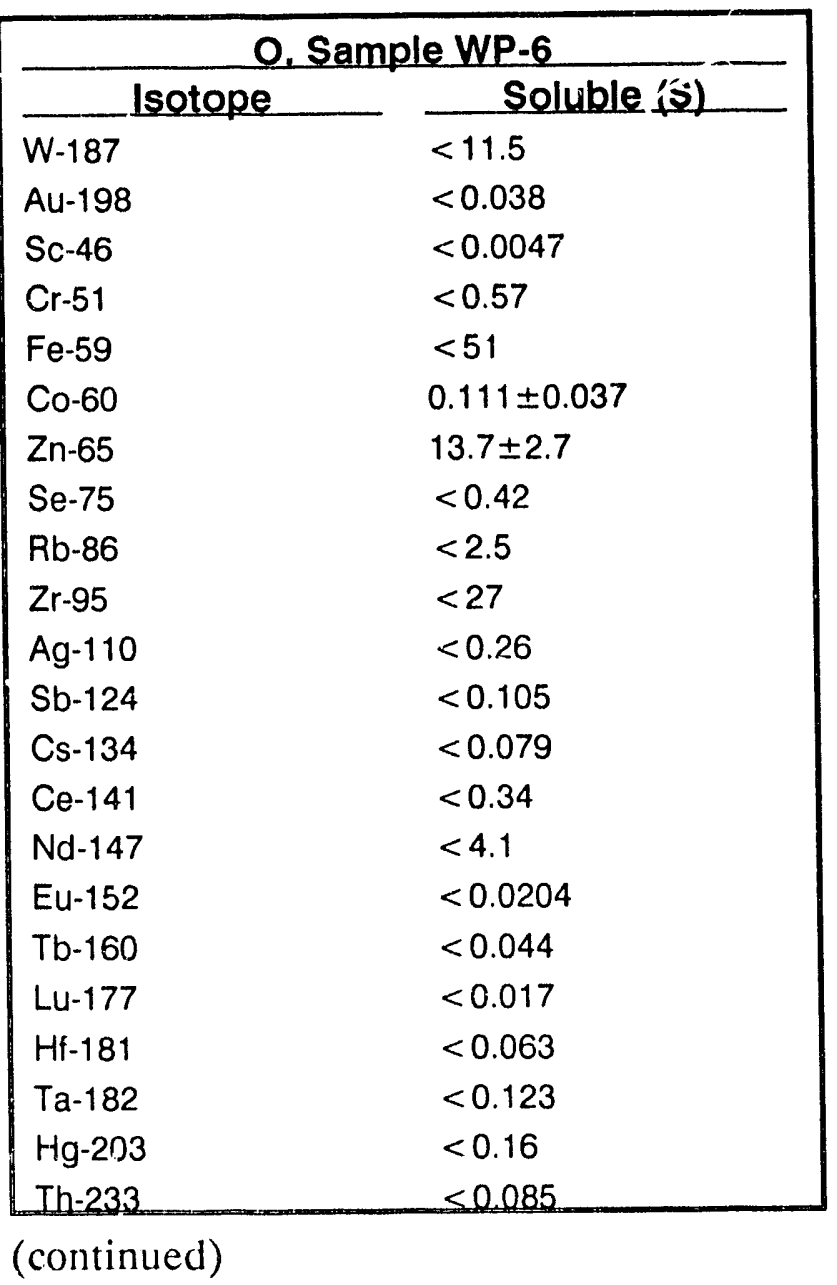


Table 3 (continued).

\begin{tabular}{|c|c|c|}
\hline & P. Sample WP-7 & \\
\hline Isotope & Soluble (S) & Clay Mineral (C) \\
\hline $\mathrm{Na}-24$ & $327400 \pm 9200$ & $14.160 \pm 410$ \\
\hline$M g-27$ & $<30000$ & $146100 \pm 6300$ \\
\hline Al-28 & $<17000$ & $50800 \pm 1900$ \\
\hline $\mathrm{Cl}-38$ & $476000 \pm 26000$ & $12830 \pm 720$ \\
\hline$K-42$ & $<46000$ & $8000 \pm 1400$ \\
\hline $\mathrm{Ca}-49$ & $<25000$ & $<940$ \\
\hline Ti-51 & $<5600$ & $3010 \pm 220$ \\
\hline$V-52$ & $<100$ & $63.7 \pm 4.2$ \\
\hline$M n-56$ & $<14$ & $229.7 \pm 8.0$ \\
\hline $\mathrm{Cu}-66$ & $<3800$ & $<300$ \\
\hline Sr-87 & $<2600$ & $<280$ \\
\hline $\ln -116$ & $<2.0$ & $<0.179$ \\
\hline $\mid-128$ & $<170$ & $<18$ \\
\hline $\mathrm{Ba}-139$ & $<1100$ & $<120$ \\
\hline Dy-165 & $<8.3$ & $4.18 \pm 0.37$ \\
\hline U-235 & $<0.015$ & $3.390 \pm 0.095$ \\
\hline Ga-72 & $<300$ & $<72$ \\
\hline As-76 & $<8.2$ & $11.2 \pm 1.1$ \\
\hline $\mathrm{Br}-82$ & $33.2 \pm 3.3$ & $3.32 \pm 0.52$ \\
\hline ino-99 & $<32$ & $<6.9$ \\
\hline La-140 & $<2.4$ & $23.06 \pm 0.97$ \\
\hline Sm-153 & $<0.18$ & $3.60 \pm 0.18$ \\
\hline$Y b-175$ & $<1.8$ & $2.22 \pm 0.14$ \\
\hline$W-187$ & $<15.3$ & $<4.0$ \\
\hline Au-198 & $<0.046$ & $0.0243 \pm 0.0050$ \\
\hline Sc- 46 & $<0.0013$ & $9.27 \pm 0.35$ \\
\hline Cr-51 & $<0.89$ & $57.1 \pm 2.7$ \\
\hline $\mathrm{Fe} \_59$ & $<77$ & $25280 \pm 940$ \\
\hline Co-60 & $<0.037$ & $9.56 \pm 0.40$ \\
\hline $\mathrm{Zn}-65$ & $<0.11$ & $5.2 \pm 1.3$ \\
\hline Se-75 & $<0.69$ & $<2.7$ \\
\hline $\mathrm{Rb}-86$ & $<3.7$ & $37.4 \pm 4.0$ \\
\hline $\mathrm{Zr-95}$ & $<40$ & $<120$ \\
\hline $\mathrm{Ag}-110$ & $<0.40$ & $<1.7$ \\
\hline Sb-124 & $<0.16$ & $<0.47$ \\
\hline Cs-134 & $<0.122$ & $3.06 \pm 0.23$ \\
\hline Ce-141 & $\leq 0.61$ & $511 \pm 1.9$ \\
\hline
\end{tabular}

(continued) 
Douglas G. Brookins

Table 3 (continued).

\begin{tabular}{|c|c|c|}
\hline \multicolumn{3}{|c|}{ P. Sample WP-7 } \\
\hline Isotope & Soluble (S) & Clay Mineral (C) \\
\hline $\mathrm{Nd}-147$ & $<6.4$ & $<8.2$ \\
\hline Eu-152 & $<0.035$ & $0.808 \pm 0.057$ \\
\hline Tb-160 & $<0.067$ & $0.748 \pm 0.099$ \\
\hline Lu-177 & $<0.027$ & $0.345 \pm 0.022$ \\
\hline$H f-181$ & $<0.098$ & $4.41 \pm 0.17$ \\
\hline Ta-182 & $<0.082$ & $0.691 \pm 0.095$ \\
\hline $\mathrm{Hg}-203$ & $<0.24$ & $<0.61$ \\
\hline Ih-233 & $<0.131$ & $7.96 \pm 0.26$ \\
\hline
\end{tabular}

\begin{tabular}{|c|c|c|}
\hline & Q. Sample WP-8 & \\
\hline Isotope & Soluble (S) & Clay Mineral (C) \\
\hline $\mathrm{Na}-24$ & $239900 \pm 6700$ & $18750 \pm 540$ \\
\hline$M g-27$ & $<26000$ & $146800 \pm 6500$ \\
\hline Al-28 & $<15000$ & $50300 \pm 2000$ \\
\hline $\mathrm{Cl}-38$ & $334000 \pm 18000$ & $19700 \pm 1100$ \\
\hline K-42 & $<40000$ & $<3700$ \\
\hline $\mathrm{Ca}-49$ & $<25000$ & $<1000$ \\
\hline Ti-51 & $<4800$ & $2900 \pm 240$ \\
\hline V-52 & $<90$ & $63.5 \pm 5.0$ \\
\hline$M n-56$ & $<11.9$ & $210.8 \pm 7.6$ \\
\hline $\mathrm{Cu}-66$ & $<3200$ & $<300$ \\
\hline Sr-87 & $<2300$ & $<270$ \\
\hline $\ln -116$ & $<1.75$ & $<0.179$ \\
\hline $\mid-128$ & $<150$ & $<18$ \\
\hline Ba-139 & $<1100$ & $<120$ \\
\hline Dy-165 & $<10.2$ & $3.28 \pm 0.33$ \\
\hline U-235 & $<0.0060$ & $3.438 \pm 0.096$ \\
\hline Ga-72 & $<280$ & $<81$ \\
\hline As-76 & $<7.8$ & $<2.2$ \\
\hline $\mathrm{Br}-82$ & $196 \pm 15$ & $4.14 \pm 0.58$ \\
\hline Mo-99 & $<29$ & $<7.0$ \\
\hline La-140 & $<2.2$ & $19.59 \pm 0.86$ \\
\hline Sm-153 & $<0.17$ & $3.33 \pm 0.16$ \\
\hline Yb-175 & $<1.6$ & $1.76 \pm 0.12$ \\
\hline$W-187$ & $<13.5$ & $<4.6$ \\
\hline $\mathrm{Au}-198$ & $<0.043$ & $<0.012$ \\
\hline Sc-46 & $<0.0059$ & $8.94 \pm 0.34$ \\
\hline Cr-51 & $<0.79$ & $53.7+2.6$ \\
\hline
\end{tabular}

(continued) 
Table 3 (continued).

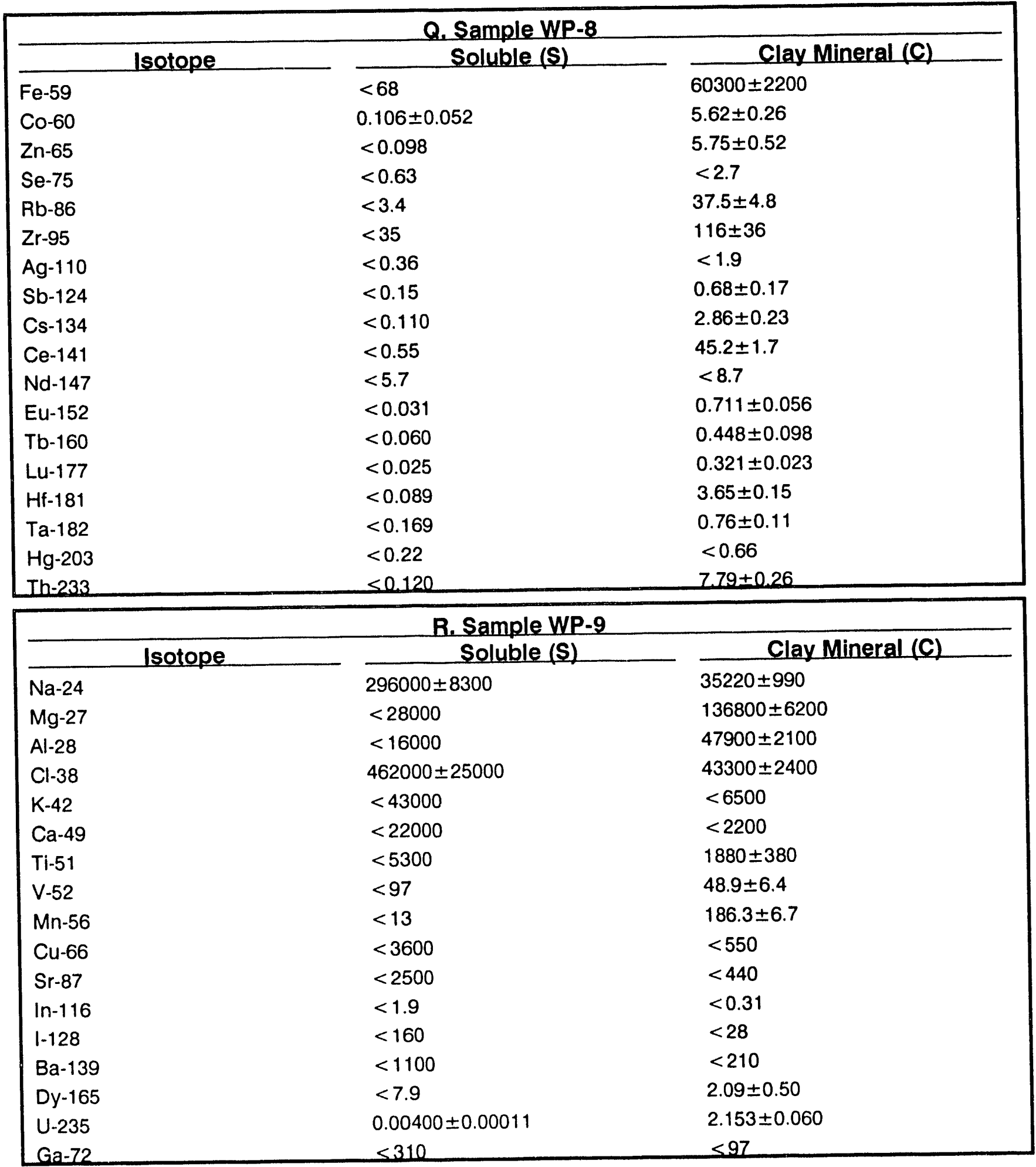

(continued) 
Douglas G. Brookins

Table 3 (continued).

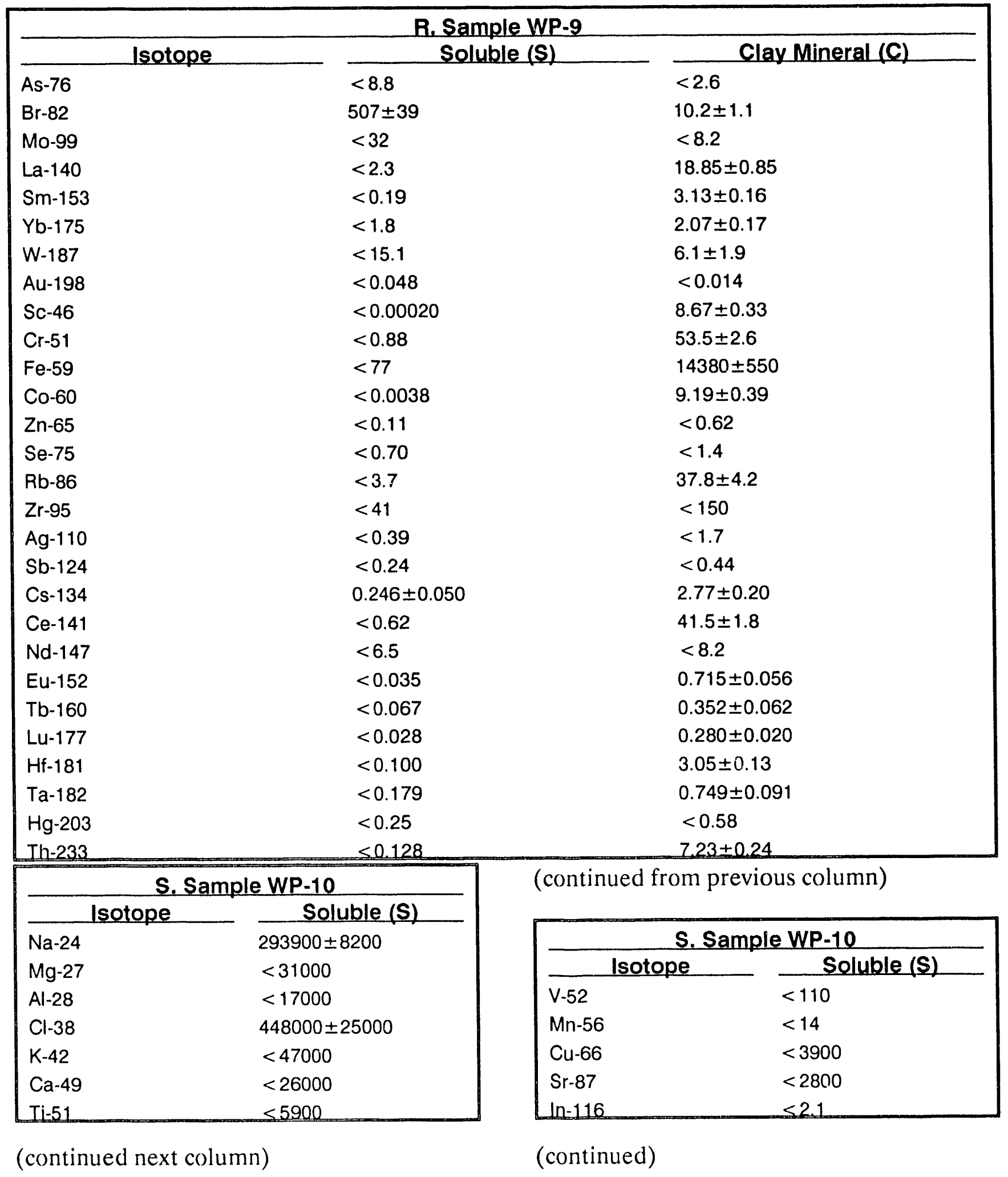


Table 3 (continued).

\begin{tabular}{|ll|}
\hline \multicolumn{2}{c|}{ S. Sample WP-10 } \\
\cline { 1 - 1 } Isotope & \multicolumn{1}{c|}{ Soluble (S) } \\
Ba-139 & $<180$ \\
Dy-165 & $<1300$ \\
U-235 & $<12.6$ \\
Ga-72 & $<0.024$ \\
As-76 & $<310$ \\
Br-82 & $<8.0$ \\
Mo-99 & $28.5 \pm 2.8$ \\
La-140 & $<30$ \\
Sm-153 & $<2.2$ \\
Yb-175 & $<0.17$ \\
W-187 & $<1.7$ \\
Au-198 & $<14.8$ \\
Sc-46 & $<0.043$ \\
Cr-51 & $<0.00019$ \\
Fe-59 & $<0.82$ \\
Co-60 & $<71$ \\
\hline
\end{tabular}

(continued from previous column)

\begin{tabular}{|c|c|}
\hline \multicolumn{2}{|c|}{ S. Sample WP-10 } \\
\hline Isotope & Soluble (S) \\
\hline $\mathrm{Zn}-65$ & $<0.10$ \\
\hline Se-75 & $<0.66$ \\
\hline $\mathrm{Rb}-86$ & $<3.5$ \\
\hline Zr-95 & $<37$ \\
\hline $\mathrm{Ag}-110$ & $<0.38$ \\
\hline $\mathrm{Sb}-124$ & $<0.15$ \\
\hline Cs-134 & $<0.114$ \\
\hline Ce-141 & $<0.58$ \\
\hline $\mathrm{Nd}-147$ & $<6.3$ \\
\hline Eu-152 & $<0.032$ \\
\hline Tb-160 & $<0.062$ \\
\hline Lu-177 & $<0.026$ \\
\hline$H f-181$ & $<0.089$ \\
\hline Ta-182 & $<0.116$ \\
\hline $\mathrm{Hg}-203$ & $<0.23$ \\
\hline Th-233 & $\leq 0124$ \\
\hline
\end{tabular}

(continued next column)

\begin{tabular}{|cc|}
\hline \multicolumn{2}{c|}{ T. Sample WP-11 } \\
\cline { 1 - 1 } Isotope & \multicolumn{1}{c|}{ Soluble (S) } \\
As-72 & $<270$ \\
Br-82 & $<6.9$ \\
Mo-99 & $52.7 \pm 4.4$ \\
La-140 & $<20$ \\
Sm-153 & $<1.97$ \\
Yb-175 & $<0.11$ \\
W-187 & $<1.42$ \\
Au-198 & $<11.8$ \\
Sc-46 & $<0.037$ \\
Cr-51 & $<0.0045$ \\
Fe-59 & $0.69 \pm 0.18$ \\
Co-60 & $<51$ \\
Zn-65 & $0.136 \pm 0.039$ \\
Se-75 & $14.2 \pm 2.7$ \\
Bb-86 & $<0.43$ \\
\hline
\end{tabular}

(continued) 
Douglas G. Brookins

Table 3 (concluded).

\begin{tabular}{|c|c|}
\hline \multicolumn{2}{|c|}{ I. Sample WP-11 } \\
\hline Isotope & Soluble (S) \\
\hline$Z r-95$ & $<26$ \\
\hline $\mathrm{Ag}-110$ & $<0.28$ \\
\hline Sb-124 & $<0.109$ \\
\hline Cs-134 & $<0.082$ \\
\hline Ce-141 & $<0.35$ \\
\hline $\mathrm{Nd}-14 \mathrm{Z}$ & $<25.3$ \\
\hline
\end{tabular}

(continued next column)

(continued from previous column)

\begin{tabular}{|ll|}
\hline \multicolumn{2}{c|}{ T. Sample WP-11 } \\
\cline { 1 - 1 } Eu-152 & \multicolumn{1}{c|}{ Soluble (S) } \\
Tb-160 & $<0.0214$ \\
Lu-177 & $<0.044$ \\
Hf-181 & $<0.019$ \\
Ta-182 & $<0.064$ \\
Hg-203 & $<0.124$ \\
Th-233 & $<0.16$ \\
& $<0.088$ \\
\hline
\end{tabular}




\section{$\mathrm{Cl} / \mathrm{Br}, \mathrm{K} / \mathrm{Rb}, \mathrm{Th} / \mathrm{U}$ Ratios}

The $\mathrm{Cl} / \mathrm{Br}$ ratios for soluble material are shown in Table 4. Values range from 750 to 21,000. The content of $\mathrm{Br}$ varies from 22.7 ppm (Sample FH40-S) to 591 ppm (WP-3-S). The seawater $\mathrm{Cl} / \mathrm{Br}$ ratio is only 280 , but in halites this ratio varies considerably. Dean (1978) has presented data indicating a range in $\mathrm{Br}$ contents of halites from $\sim 20$ to $400 \mathrm{ppm}$ and, for a theoretical Na percentage of 38 , the range in halite $\mathrm{Cl} / \mathrm{Br}$ is from 950 to $19,000 . \mathrm{Br}$ tends to concentrate in the evaporite brines well above its seawater concentration of 67 ppm under certain conditions, and minerals

Table 4. $\mathrm{Cl} / \mathrm{Br}$ Ratios

\begin{tabular}{|c|c|c|c|}
\hline \multirow{2}{*}{\multicolumn{2}{|c|}{$\frac{\text { Sample }}{\text { Soluble Fractlons* }}$}} & \multirow[t]{2}{*}{ Sample } & \multirow[t]{2}{*}{$\mathrm{Cl} / \mathrm{Br}$} \\
\hline & & & \\
\hline WP-1-S & 2900 & WP-11-S & 9200 \\
\hline WP-2-S & 15000 & $\mathrm{FH}-4-\mathrm{S}$ & 6900 \\
\hline WP-3-S & 750 & FH6A-S & 17000 \\
\hline WP-4-S & 1900 & FH6B-S & 4000 \\
\hline WP-5-S & 6200 & FH26-S & 7100 \\
\hline WP-6-S & - & $\mathrm{FH} 27-\mathrm{S}$ & 1100 \\
\hline WP-7-S & 14000 & FH32-S & 7100 \\
\hline WP-8-S & 1700 & FH40-S & 21000 \\
\hline WP-9-S & 910 & FH42-S & 3600 \\
\hline \multirow[t]{2}{*}{ WP-10-S } & 16000 & FH45-S & 4300 \\
\hline & & $\begin{array}{l}\text { Avg. sea- } \\
\text { water }\end{array}$ & 280 \\
\hline \multicolumn{4}{|c|}{ Whole Rocks, FH Series } \\
\hline FH4-WR & 10000 & FH32-WR & 10000 \\
\hline FH6B-WR & 7200 & FH40-WR & 8500 \\
\hline $\mathrm{FH} 26-W R$ & 11000 & $\mathrm{FH} 42-\mathrm{WR}$ & 8600 \\
\hline $\mathrm{FH} 27-\mathrm{WR}$ & 13000 & FH45-WR & 10000 \\
\hline
\end{tabular}

such as sylvite and carnallite incorporate high amounts of Brinto their structures, leaving the halite somewhat depleted in $\mathrm{Br}$. The range in $\mathrm{Cl} / \mathrm{Br}$ ratio for the soluble material (Table 3 ) is not considered anomalous in terms of $\mathrm{Cl} / \mathrm{Br}$, but some samples (WP-10-S, FH6A-S, FH40$\mathrm{S})$ do contain very low $\mathrm{Br}$ contents.

The $\mathrm{Cl} / \mathrm{Br}$ ratios of whole-rock samples range from 7200 to 13,000 , well within the range of normal halites (Dean, 1978). This is not surprising, because in most samples the amount of nonhalide material is small.

The $\mathrm{Cl} / \mathrm{Br}, \mathrm{K} / \mathrm{Rb}$, and $\mathrm{Th} / \mathrm{U}$ ratios for clay minerals and insoluble clay-oxyhydroxide mixtures are shown in Table 5 . The range in $\mathrm{Cl} / \mathrm{Br}$ ratios is from 260 to 6500 . The $\mathrm{Cl} / \mathrm{Br}$ ratio for average shale (representing "typical" marine clay minerals) is only 34 ; hence the data in Table 5 show either excess $\mathrm{Cl}$ or somewhat depleted $\mathrm{Br}$ contents with respect to the Table 5. $\mathrm{Cl} / \mathrm{Br}, \mathrm{K} / \mathrm{Rb}$, and $\mathrm{Th} / \mathrm{U}$ Ratios for Insoluble Residues and Clay Separates

\begin{tabular}{|c|c|c|c|}
\hline Sample* & $\mathrm{Cl} / \mathrm{Br}$ & K/Bb & Th/U \\
\hline WP-1-C & 2000 & 160 & 2.7 \\
\hline WP-2-C & 1600 & 110 & 2.8 \\
\hline WP-3-C & 5000 & 240 & 2.9 \\
\hline WP-4-C & 6500 & 160 & 2.2 \\
\hline WP-7-C & 3900 & 210 & 2.4 \\
\hline WP-8-C & 4800 & - & 2.3 \\
\hline WP-9-C & 4200 & - & 3.4 \\
\hline FH4-I & 1500 & - & 2.8 \\
\hline FH6B-I & 260 & 150 & 3.4 \\
\hline $\mathrm{FH} 27-\mathrm{I}$ & 56000 & - & 2.2 \\
\hline $\mathrm{FH} 32-\mathrm{I}$ & 1400 & 200 & 1.9 \\
\hline $\mathrm{FH} 42-\mathrm{I}$ & 2600 & 120 & 1.9 \\
\hline $\mathrm{FH} 45-\mathrm{I}$ & 2100 & 180 & 3.6 \\
\hline Avg. shale & 34 & 180 & 3.4 \\
\hline
\end{tabular}

*IaInsoluble Residue; Ca Clay Separate 
averages for shale. We attribute the high $\mathrm{Cl} / \mathrm{Br}$ ratios of the samples to (1) small amounts of halide minerals present in the hand-picked clay mineral-oxyhydroxide mixtures, and (2) incomplete removal of soluble salts in the insoluble fractions.

The $\mathrm{K} / \mathrm{Rb}$ ratios, however, are less sensitive to such contamination. The ratios vary from 110 to 240 , with a mean of 170 . This value is very close to that of average shale of 178 (Table 5). The relevance of this is discussed below.

$\mathrm{Th} / \mathrm{U}$ ratios for the same samples range from 1.9 to 3.6 (Table 5) with a mean of 2.7 , which is comparable to the average $\mathrm{Th} / \mathrm{U}$ ratio for shale of 3.43. Both the Th and $U$ contents of these samples (Table 3 ) are lower than for average shale (i.e., $12 \mathrm{ppm}$ Th, $3.5 \mathrm{ppm} \mathrm{U}$ ). This is probably due to simple dilution of the Th- and U-bearing phases with Th- and Upoor material. These data indicate that uranium has apparently not been leached from these samples, as discussed below.

\section{Studies of Rare-Earth Elements}

Rare-earth element (REE) studies are of relevant to determining the integrity of minerals such as clays in an evaporitic environment, because the $\mathrm{REE}$ can be mobilized in $\mathrm{Cl}-\mathrm{SO}_{4}$ $\mathrm{CO}_{3}$ environments (Brookins, 1983), and depletions of total REE will result. Also, more subtle effects of the differences in light REE (LREE) versus heavy REE (HREE) can be correlated with the amount of interaction of the clay minerals with the brine. Furthermore, the REE studies represent an independent check on the K-Ar geochronology and other tests for the stability of clay minerals.
Following the convention of Haskin and Schmitt (1967), the REE data of Table 3 have been normalized to the analogous REE daia for chondrite meteorites (CHON) lising the new values recommended by Anders and Ebihara (1982). Also following the convention, the ratios of lanthanide concentrations in samples to those in chondrite meteorities

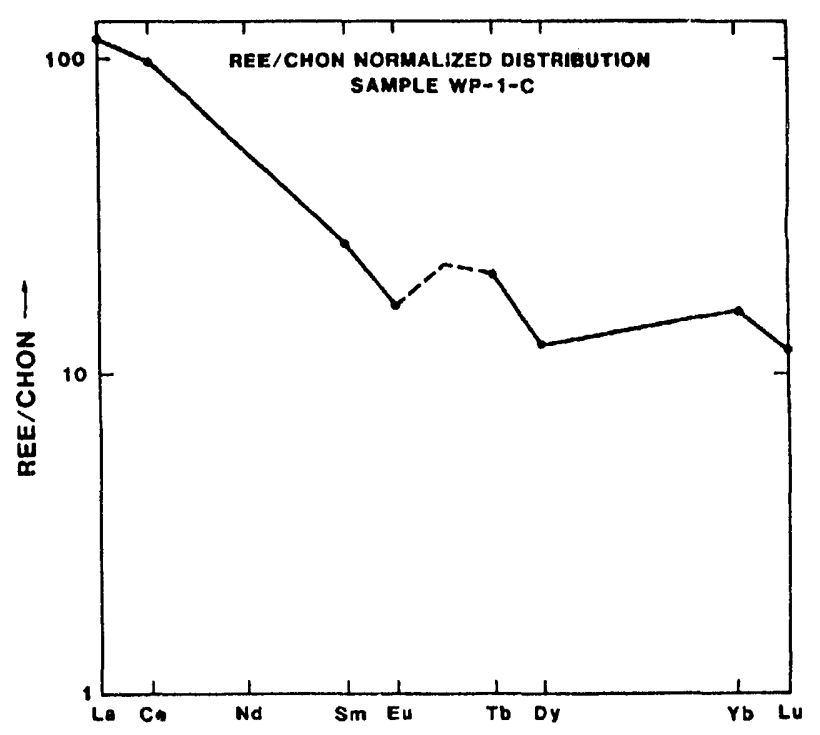

Figure 1. REE/CHON Normalized Distribution, Sample WP-1-C

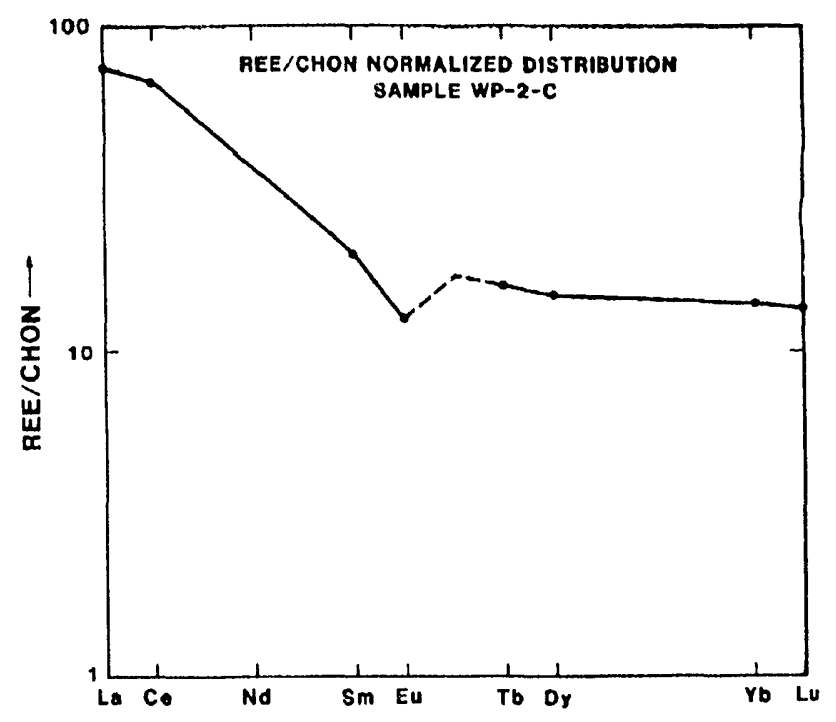

Figure 2. REE/CHON Normalized Distribution, Sample WP-2-C 


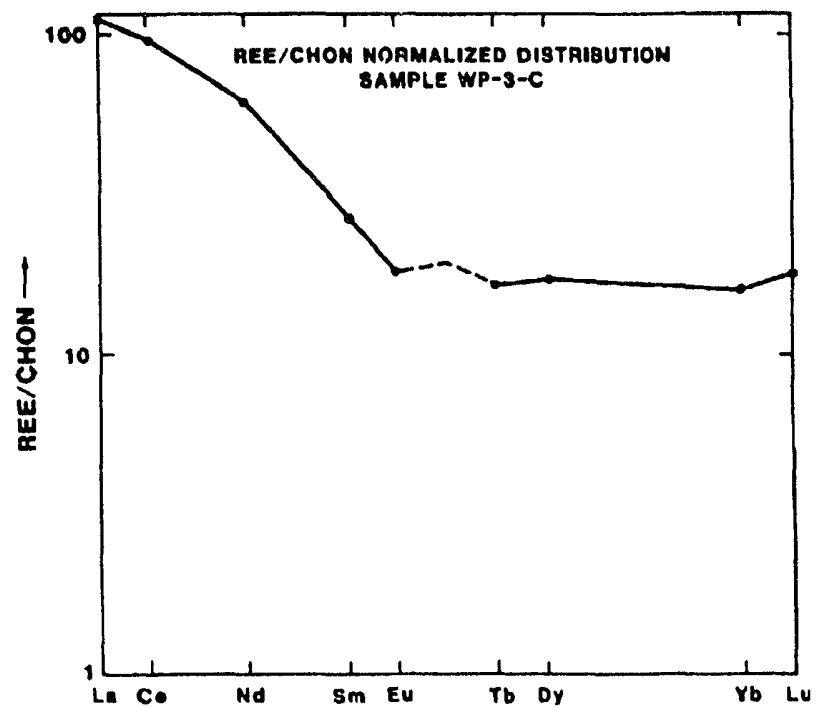

Figure 3. REE/CHON Normalized Distribution, Sample WP-3-C

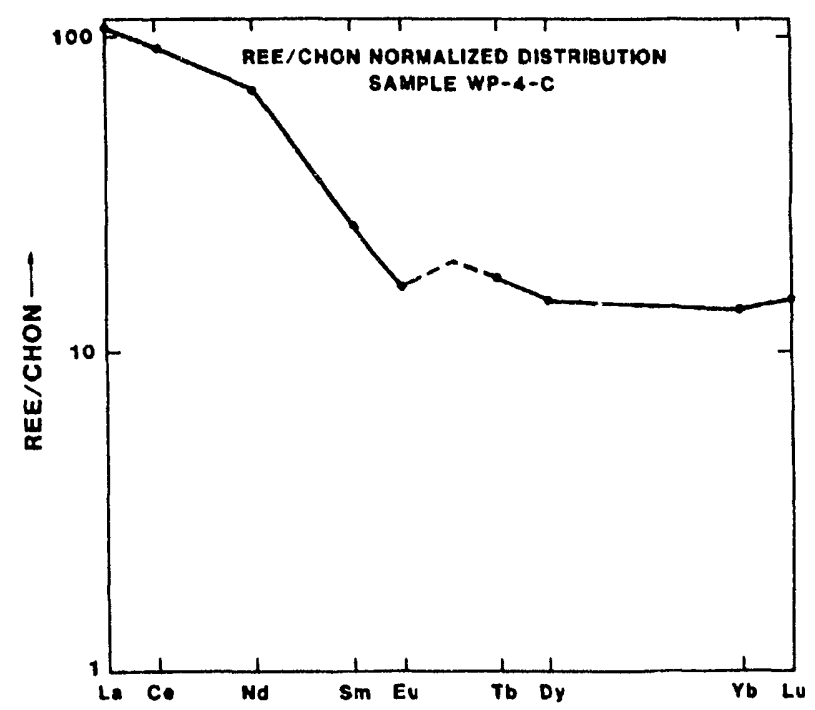

Figure 4. REE/CHON Normalized Distribution, Sample WP-4-C

( $\mathrm{REE} / \mathrm{CHON}$ ) were plotted versus relative position of the REE from $\mathrm{La}$ through $\mathrm{Lu}$ in order of increasing atomic number. The individual plots are shown in Figures 1 through 8 for the WP samples and in Figures 9 through 14 for the FH samples. The samples represent either clay mineral-oxyhydroxide separates (C) or insoluble residues (I) except for WP-5-

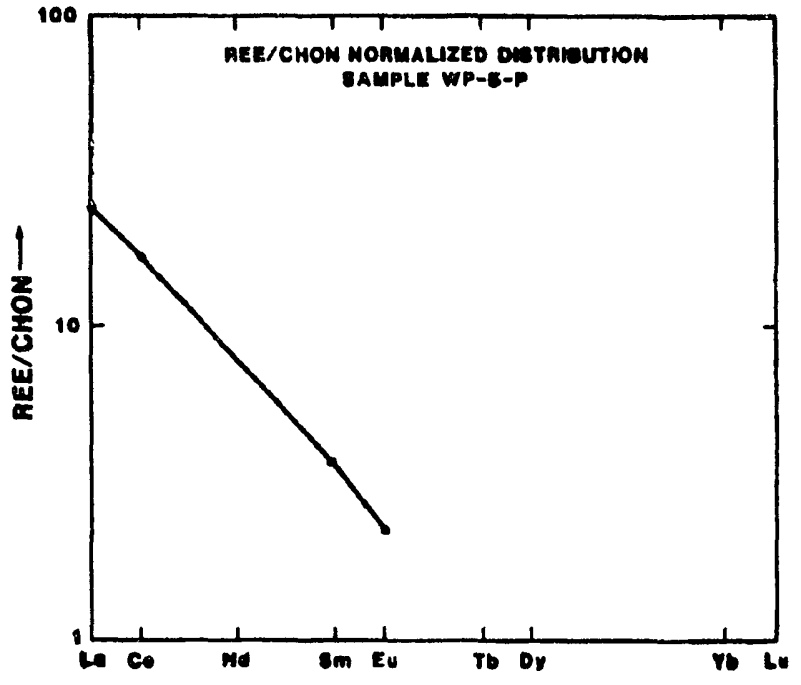

Figure 5. REE/CHON Normalized Distribution, Sample WP-5-P

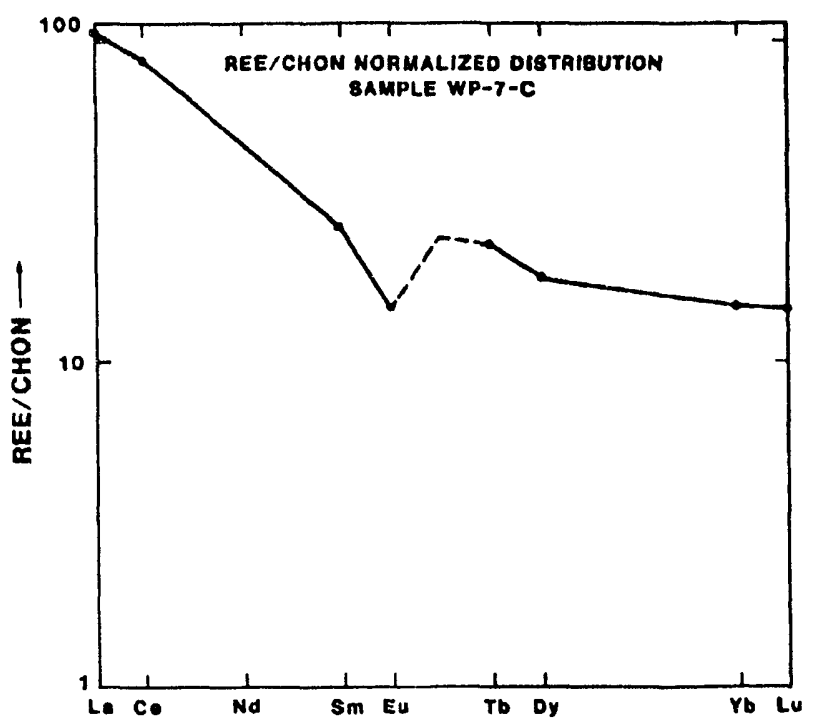

Figure 6. REE/CHON Normalized Distributions, Sample WP-7-C

$\mathrm{P}$, which is a hand-picked polyhalite sample (Figure 5).

Most samples show moderate enrichment in the LREE, as is consistent with studies of clay minerals from many locations (see Brookins, 1979, for discussion). In the absence of $\mathrm{Gd}$ analyses, the line connecting the $\mathrm{Sm}$ and $\mathrm{Tb}$ points is projected as a dashed line from the 


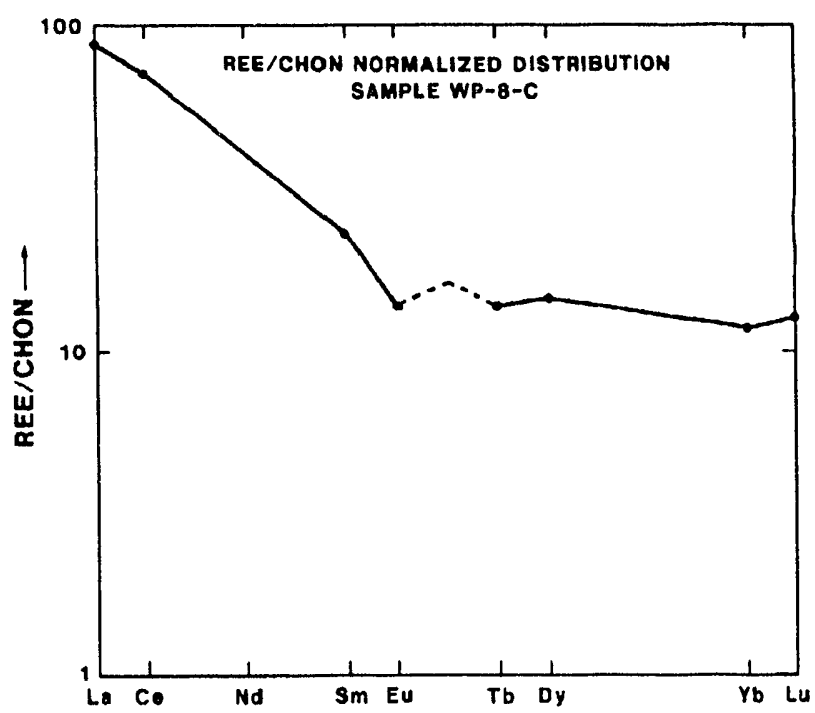

Figure 7. REE/CHON Normalized Distribution, Sample WP-8-C

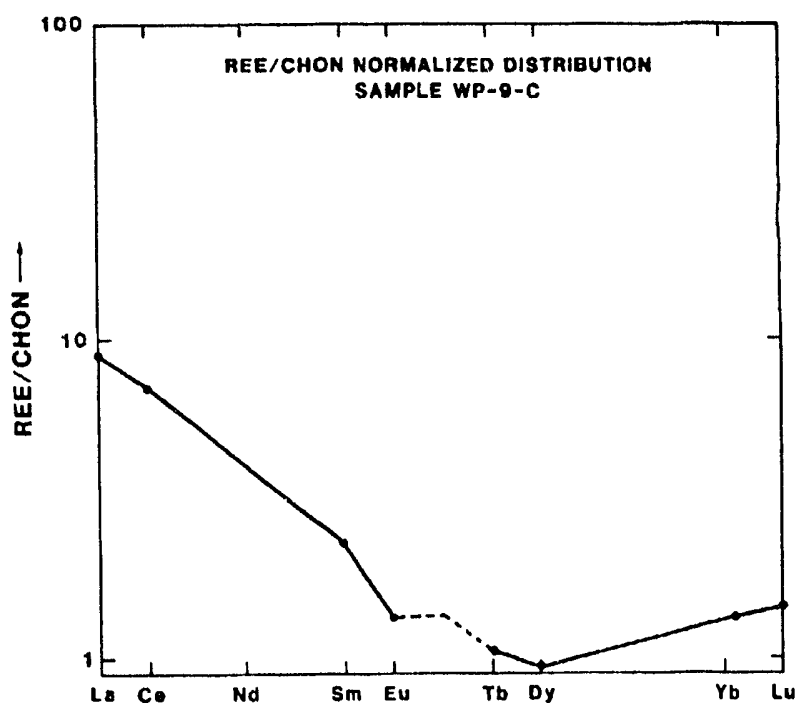

Figure 8. REE/CHON Normalized Distribution, Sample WP-9-C

Tb point to the position corresponding to $\mathrm{Gd}$ (midway between $\mathrm{Eu}$ and $\mathrm{Tb}$ on the horizontal coordinate); the dashed line connecting the Eu and projected Gd points approximately defines the HREE end of the Eu anomaly (i.e., selective exclusion of $\mathrm{Eu}$ from minerals other than feldspar, where it concentrates as the divalent, rather than trivalent cation). The Eu anomaly is consistent with a normal-type

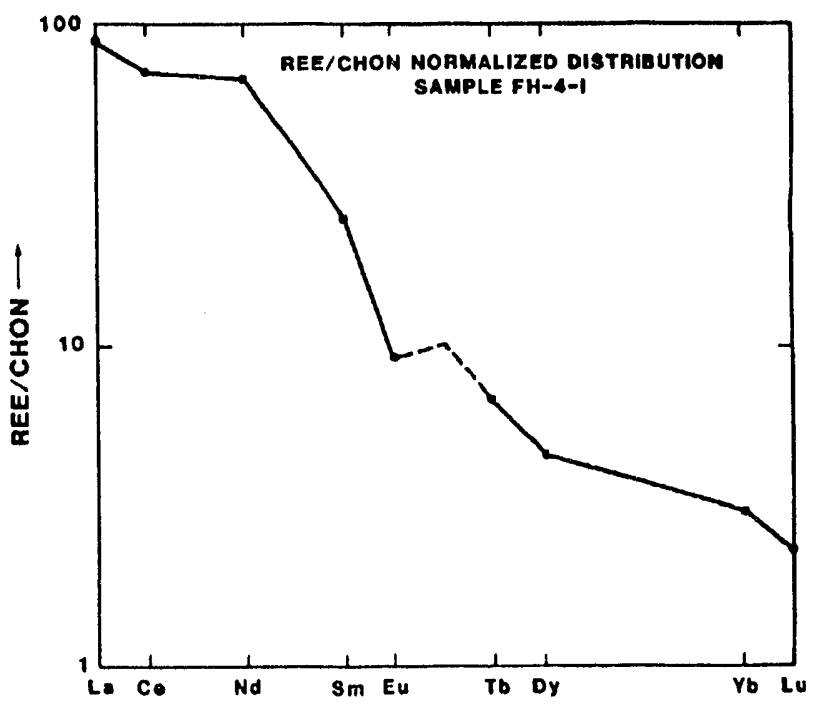

Figure 9. REE/CHON Normalized Distribution, Sample $\mathrm{FH}-4-\mathrm{I}$

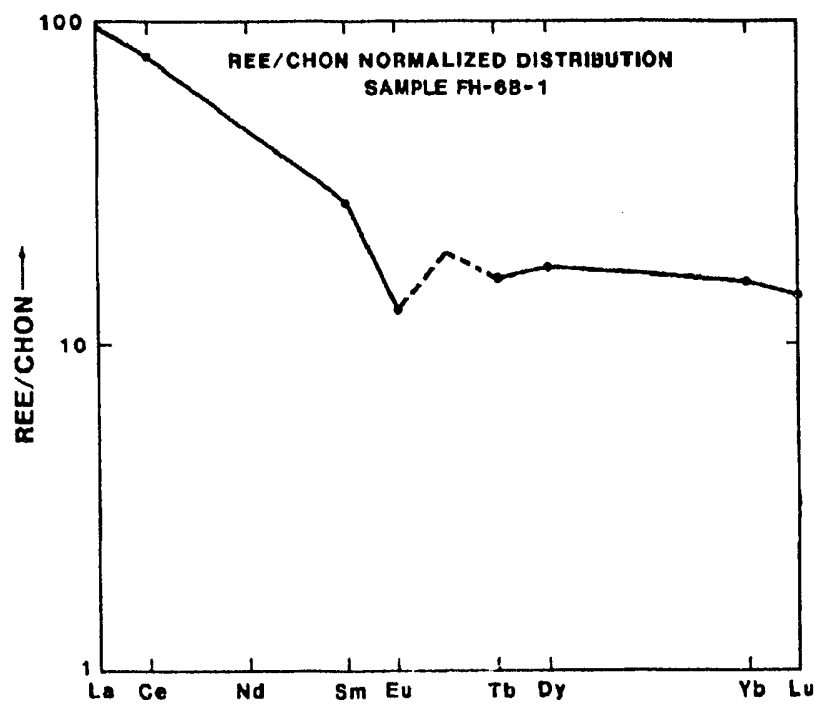

Figure 10. REE/CHON Normalized Distribution, Sample FH-6B-I

LREE distribution for clays and for shales. At the HREE end of the curves, however, there is a pronounced tendency for the curves to flatten out, as is commonly noted in many clays. Any hypothetical scenario of REE exchange with an evaporitic brine should take into account the very small REE content ot seawater, and thus of brines formed by evaporation (Elderfield and Greaves, 1982). Sev- 


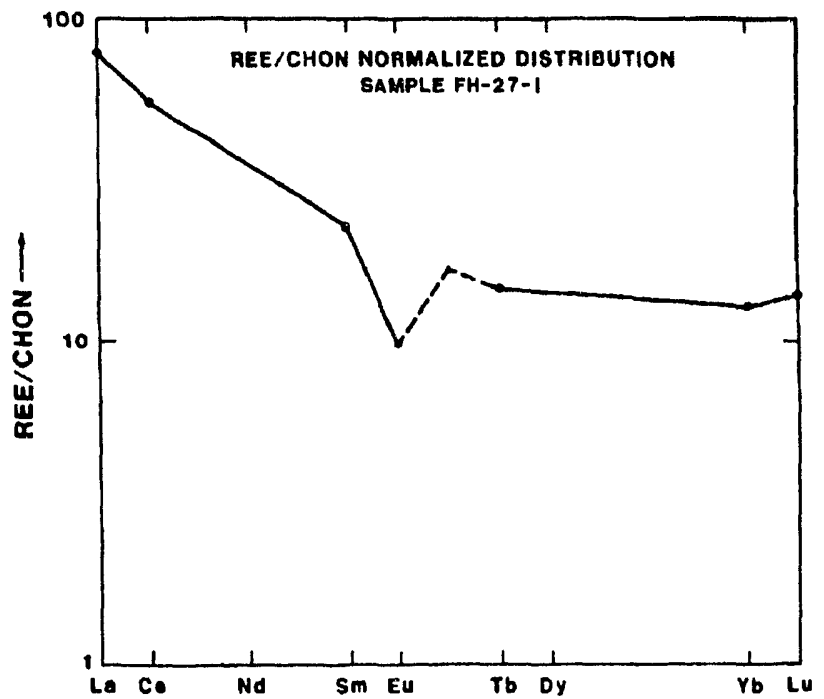

Figure 11. REE/CHON Normalized Distribution, Sample FH-27-I

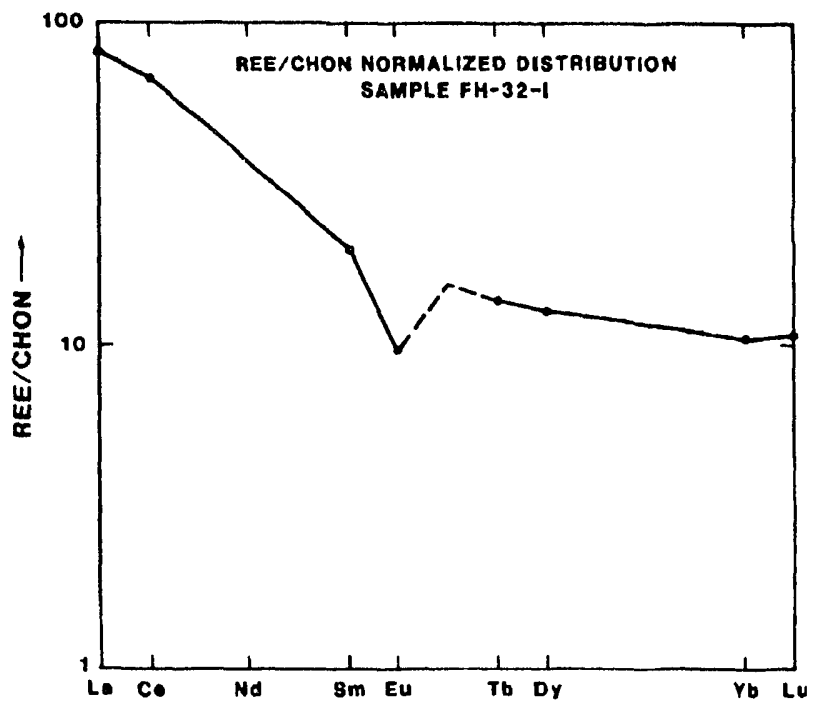

Figure 12. REE/CHON Normalized Distribution, Sample FH-32-I

eral types of effects of interactions between REE and evaporite-brine are possible. Such interactions include an overall depletion of the REE because of removal into the brines, and preferential loss of HREE as a result of the effects of $\mathrm{Mg}$-exchange between brine and clay mineral. Only sample FH-4-I (Figure 9) shows a pronounced HREE downward trend, and none of the other clay-mineral-rich sam-

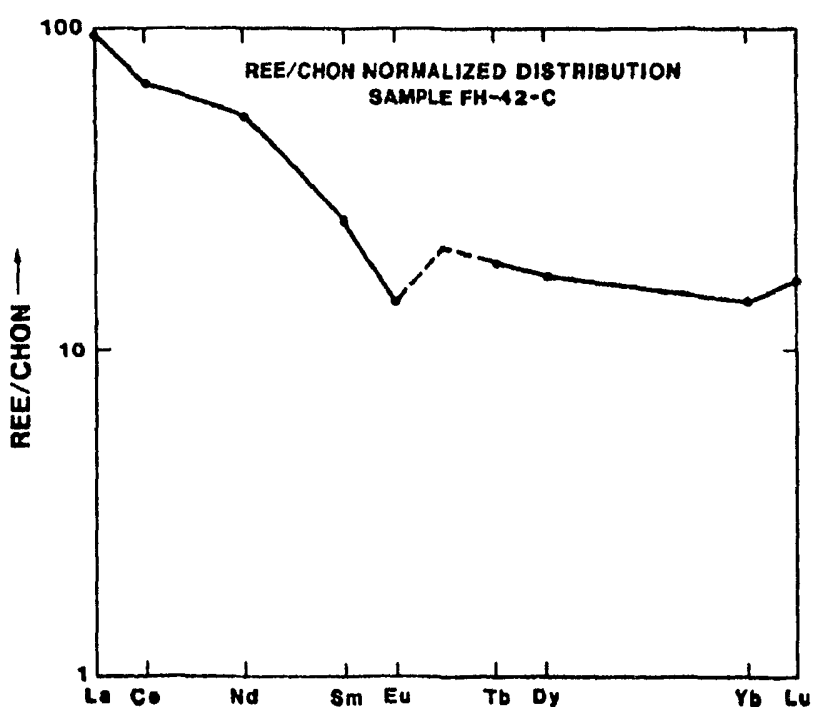

Figure 13. REE/CHON Normalized Distribution, Sample FH-42-C

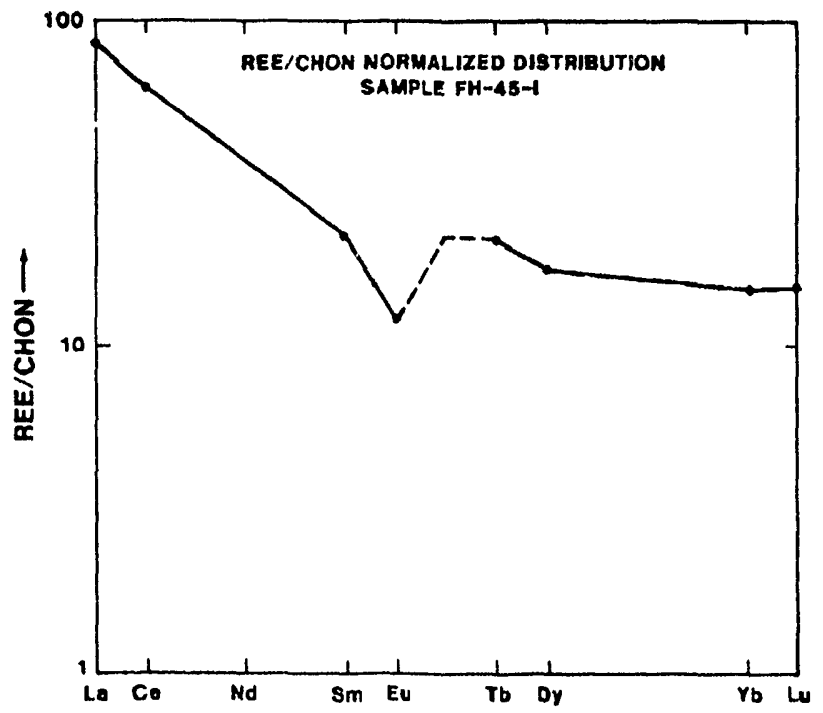

Figure 14. REE/CHON Normalized Distribution, Sample FH-45-I

ples $(\mathrm{C}, \mathrm{I})$ reflect HREE loss. In fact, the flattening of the curves could suggest HREE addition except that there is no source for such addition. In the case of the polyhalite, WP-5-P (Figure 5), the curve reflects the preferential enrichment of the LREE (i.e., in the Ca sites) versus HREE. 
Douglas G. Brookins

The REE data for the clay minerals strongly suggest that residence in the evaporitic brines was not sufficient to affect the REE abundance distribution in the clay minerals. This is important because it dernonstrates the ability of such clay- oxyhydroxide materials to serve as getters for the lanthanide REE, which may act as analogues for actinides.

Table 6. K-Ar Age Determinations

\section{K-Ar Dates}

$\mathrm{K}$-Ar dates were obtained by Geochron Laboratories of Cambridge, Massachusetts. Table 6 contains the data for radiogenic argon $\left({ }^{*} \mathrm{Ar}\right)$, in ppm, total $\mathrm{K}$ in $\%$, ratio of radiogenic Ar to total $\mathrm{Ar}\left({ }^{*} \mathrm{Ar} / \varepsilon{ }^{40} \mathrm{Ar}\right.$, to indicate

\begin{tabular}{|c|c|c|c|c|}
\hline Sample & ${ }^{* 40} \operatorname{Ar}(p p m)$ & $K(\%)$ & ${ }^{40} \mathrm{Ar} / \varepsilon^{40} \mathrm{Ar}$ & $\pm(\mathrm{Ma})$ \\
\hline \multirow[t]{3}{*}{$\mathrm{FH}-4-\mathrm{I}$} & 0.005578 & 0.634 & 0.243 & $114 \pm 5$ \\
\hline & 0.004565 & 0.684 & 0.173 & \\
\hline & 0.006156 & - & 0.195 & \\
\hline \multirow[t]{3}{*}{$F H-6 A-I$} & 0.09240 & 6.687 & 0.894 & $187 \pm 7$ \\
\hline & 0.1056 & 7.245 & 0.795 & \\
\hline & 0.08967 & 6.987 & 0.790 & \\
\hline \multirow[t]{2}{*}{$\mathrm{FH}-6 \mathrm{~B}-\mathrm{C}$} & 0.02195 & 0.0694 & 0.387 & $378 \pm 15$ \\
\hline & 0.02007 & 0.741 & 0.526 & \\
\hline \multirow[t]{2}{*}{$\mathrm{FH}-26-\mathrm{C}$} & 0.02783 & 0.931 & 0.548 & $382 \pm 15$ \\
\hline & 0.02516 & 0.860 & 0.492 & \\
\hline \multirow{2}{*}{$\mathrm{FH}-27-\mathrm{C}$} & 0.02315 & 0.821 & 0.659 & $365 \pm 14$ \\
\hline & 0.02259 & 0.806 & 0.546 & \\
\hline \multirow[t]{2}{*}{$\mathrm{FH}-27-1(\mathrm{P})$} & 0.001913 & 0.491 & 0.125 & $55.5 \pm 3$ \\
\hline & 0.001873 & 0.475 & 0.138 & \\
\hline \multirow[t]{4}{*}{$\mathrm{FH}-27-\mathrm{WR}$} & 0.000898 & 0.045 & 0.164 & $324 \pm 20$ \\
\hline & 0.000555 & 0.050 & 0.193 & \\
\hline & 0.002236 & 0.057 & 0.415 & \\
\hline & 0.001311 & - & 0.334 & \\
\hline \multirow[t]{2}{*}{$\mathrm{FH}-32-\mathrm{C}$} & 0.02272 & 0.769 & 0.714 & $387 \pm 15$ \\
\hline & 0.02305 & 0.757 & 0.555 & \\
\hline \multirow{2}{*}{$F H-42-I$} & 0.005177 & 0.147 & 0.053 & $88.7 \pm 5.3$ \\
\hline & 0.001335 & 0.197 & 0.087 & \\
\hline \multirow[t]{3}{*}{$\mathrm{FH}-45-\mathrm{C}$} & 0.02755 & 0.904 & 0.753 & $390 \pm 15$ \\
\hline & 0.02583 & 0.860 & 0.657 & \\
\hline & - & 0.273 & & \\
\hline \multicolumn{5}{|c|}{$\begin{array}{l}\text { Ma-millions of years before the present, based on the mean of all replicates } \\
\text { C-clay mineral separate } \\
\text { I-insoluble residue } \\
\text { O- oxyhydroxide minerals } \\
\text { P-polyhalite }\end{array}$} \\
\hline
\end{tabular}


magnitude of air correction), and $\mathrm{K} / \mathrm{Ar}$ age in millions of years before the present (Ma). The ages as reported by Geochron have been corrected to the most recently available decay constants for ${ }^{40} \mathrm{~K}$. Background information on the $\mathrm{K}$-Ar dating of the WIPP and related evaporites and associated materials may be found in Brookins et al (1980), Register and Brookins (1980), and Brookins (1981; 1982).

Some earlier work indicated that primary evaporite minerals such as sylvite and polyhalite retained radiogenic ${ }^{40} \mathrm{Ar}$ well enough to give reliable dates, many of which clustered in the age range 180 to $220 \mathrm{Ma}$. Brookins et al. (1980) showed that an empirical correction factor could be applied to polyhalites for admixed halide minerals (i.e., halide minerals lose ${ }^{* 40} \mathrm{Ar}$ easily) with resultant corrected ages $>200$ Ma. Register (1981; see also Register and Brookins, 1980) examined the $\mathrm{Rb}-\mathrm{Sr}$ Systematics of clay minerals form the WIPP area evaporites, with a resultant isochron date of $390 \pm 77 \mathrm{Ma}$. Brookins et al. (1980) and Brookins (1981) interpreted this result as evidence that the internal $\mathrm{Rb}-\mathrm{Sr}$ systematics of clay minerals, presumably of aeolian origin, had not been aitered to reflect the age of evaporites (e.g., 220 to $230 \mathrm{Ma}$ ) during entrapment in the evaporite mother-liquor.

The present study emphasizes clay minerals from the WIPP underground workings. Two types of samples were used for study; those from which enough clay and oxyhydroxide minerals could be mechanically separated, and those in which an attempt was made to separate these minerals by dissolution of halite. One sample (WP-5) also contained enough polyhalite for hand separation.

The attempt at concentrating sufficient uncontaminated insoluble material by dissolution was not successful. The $x$-ray diffraction data (Table 2) show that the samples so treated contain moderate amounts of gypsum, halite, and anhydrite; their chemistry (Table 3) reflects the presence of significant amounts of $\mathrm{K}$, presumably derived from the evaporitic salts. This excess $\mathrm{K}$ is thought to be the prime reason for the wide range (55 to $114 \mathrm{Ma}$ ) in the $\mathrm{K}-\mathrm{Ar}$ dates for the insoluble residues (Table 6). No geological significance can be attributed to these dates.

However, the clay minerals (with or without oxyhydroxide minerals) separated by hand yield dates from 365 to $390 \mathrm{Ma}$, in agreement with the Rb-Sr work of Register and Brookins (1980). These dates are offered as evidence that the K-Ar systematics of these clay minerals were not reset to the age of evaporitic mineral formation when these minerals were entrapped in the evaporitic brine environment. The significance of this conclusion is discussed below.

One hand-picked polyhalite sample (FH6A) yields a date of $187 \pm 7 \mathrm{Ma}$. Data are not available for the $\mathrm{Na}$ content of the sample; hence, a correction (e.g., Brookins et al., 1980) cannot be applied; hence, it is not known if this polyhalite would yield a corrected date of 210 to 230 Ma like the other samples from the area.

$\mathrm{K}$-Ar dating was attempted on one sample of clay-bearing halite (FH27). Surprisingly, it yielded an apparent K-Ar date of $324 \pm 20$ $\mathrm{Ma}$. This age may reflect the presence of the $365 \mathrm{Ma}$ clay in the sample (FH27-C), diluted with polyhalite and halite.

\section{Rb-Sr Dates}

$\mathrm{Rb}-\mathrm{Sr}$ dates were determined in the University of New Mexico Geology Department Geochronology Laboratory. Samples were 
Douglas G. Brookins

Table 7. Rb/Sr Geochronologic Data

\begin{tabular}{|c|c|c|c|c|}
\hline Sample $^{1}$ & $\mathrm{Rb}$ (ppm) & $\operatorname{sr}(p, p m)^{2}$ & ${ }^{87} \mathrm{Sr}^{86} \mathrm{Sr}^{2}$ & $\left.{ }^{87} \mathrm{Rb}\right|^{80} \mathrm{Sr}$ \\
\hline $\mathrm{FH} 4-\mathrm{I}$ & 10.96 & $9041.0(1)$ & $0.7072(8)$ & 0.00351 \\
\hline FH6A-I & 0.54 & $3481.7(1)$ & $0.7076(3)$ & 0.00045 \\
\hline FH6B-I & 42.18 & $58.3(6)$ & $0.7199(9)$ & 2.0937 \\
\hline FH26-WR & 0.15 & $1.2(2)$ & $0.7083(0)$ & $0.355 \%$ \\
\hline $\mathrm{FH} 27-\mathrm{I}$ & 2.55 & $7449.1(4)$ & $0.7080(5)$ & 0.00099 \\
\hline FH32-WR & 0.45 & $1.7(6)$ & $0.7130(0)$ & 0.74016 \\
\hline FH10-I & 11.41 & $4179.5(3)$ & $0.7073(5)$ & 0.00799 \\
\hline $\mathrm{FH} 42-\mathrm{I}$ & 40.57 & $83.7(3)$ & $0.7159(9)$ & 1.40306 \\
\hline $\mathrm{FH} 45-\mathrm{I}$ & 48.17 & $42.0^{\prime}(2)$ & $0.7271(0)$ & 3.32312 \\
\hline WP-1-I & 46.31 & $25.1(4)$ & $0.7405(7)$ & 5.34694 \\
\hline WP-2-1 & 36.77 & $33.0(0)$ & $0.7759(2)$ & 3.22964 \\
\hline
\end{tabular}

1. I-insolublc; WR - whole rock

2. Icast significant digit is parenthesized

prepared using standard dissolution metiods for siiicate-oxide assemblages, involving dissolution in HF:deionized water, cooling, filrering, and separation of $\mathrm{Rb}$ and $\mathrm{Sr}$ by ion exchange chromatography with $2.2 \mathrm{~N} \mathrm{HCl}$ as the eluant. Samples were spiked with ${ }^{84} \mathrm{Sr}$ and ${ }^{87} \mathrm{Rb}$ spikes before dissolution. After the ion exchange procedure, samples were fused in quartz beakers, cooled, and stored. Samples were then loaded onto rhenium filaments, and isotopic data were obtained using a Nier-design thermal ionization mass spectrometer. The data are presented in Table 7 and plotted in Figure 15.

Table 7 shows total $\mathrm{Rb}$ and $\mathrm{Sr}$ in ppm and the ${ }^{87} \mathrm{Sr} /{ }^{86} \mathrm{Sr}$ isotopic ratio. The total $\mathrm{Rb}$ and $\mathrm{Sr}$ data are precise to $\pm 0.25 \%(10)$ of the reported value, and the reported ${ }^{87} \mathrm{Sr} /{ }^{86} \mathrm{Sr}$ ratios are precise to $\pm 0.00002(2 \sigma)$. The isochron in Figure 15 was calculated using five iterations of the least-squares method of York (196!). The regression of the data in Figure 15 yielas a $\mathrm{Rb}-\mathrm{Sr}$ isochron date of $428 \pm 7 \mathrm{Ma}$ with initial ${ }^{87} \mathrm{Sr} /{ }^{86} \mathrm{Sr}=0.70707 \pm 0.00013$.
K-Ar dates for clay minerals (discussed above) are tightly clustered between $\sim 360$ and $390 \mathrm{Ma}$. The insoluble residues from the samples of this study define a fairly good isochron with a date of $428 \mathrm{Ma}$. 'This date is within reasonable agreement with previously reported $\mathrm{K}-\mathrm{Ar}$ and $\mathrm{Rb}-\mathrm{Sr}$ dates (see above).

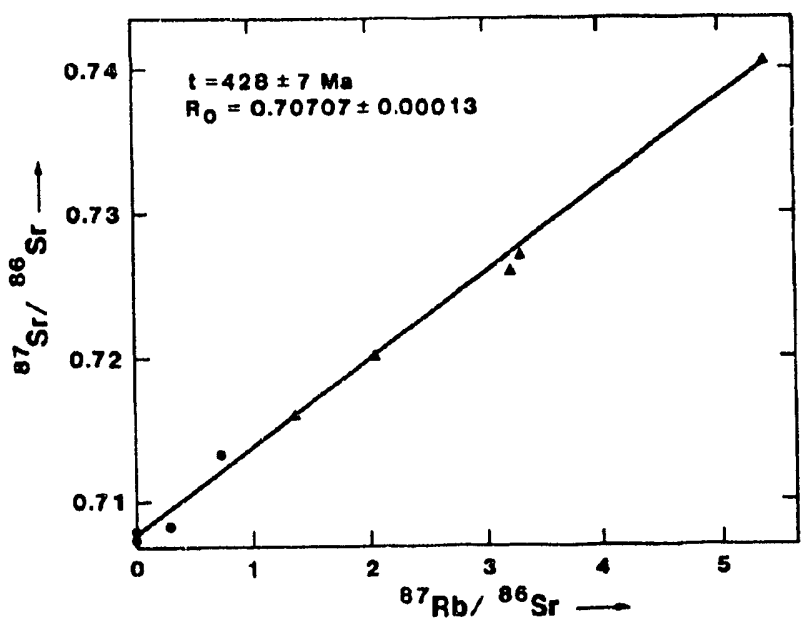

Figure 15. Rb-Sr Isochron for Clay Minerals and Oxyhydroxide Minerals 
The original interpretation of these pre-Permian dates, by Brookins et al (1980) and Brookins (1981) was that the clay minerals were of aeolian origin, and that their K-Ar and $\mathrm{Rb}$-Sr isotopic systematics had not been reset upon incorporation into and reaction with the evaporitic brines. The data defining the $390 \pm$ $77 \mathrm{Ma} \mathrm{Rb}-\mathrm{Sr}$ isochron date for clay minerals fron. $₫ 3$ veral locations (Register, 1981) were more scattered than those in the present study, and no quantitative comments could be made concerning provenance and thus extent of diagenetic reaction. The linear isochron of Figure 15, however, is a much better fit (i.e., the error on the age is only $7 \mathrm{Ma}$, and the error on the initial intercept only \pm 0.00013 ). Because the samples used for this study are all from the WIPP site, problems such as mixed provenance may have been avoided. For example, if samples with different prehistories, including different initial ratios, are homogenized at some time, then several parallel isochrons all of the same age may result (Brookins, 1985). This possibility cannot be dismissed for the samples reported by Register (1981). The data of this study also suggest, but do not unequivocally prove, that there may have been a $400-$ to 450 -Ma source area for the clay minerals that dominated the aeolian input. This is surprising, because scurce areas theoretically could have included everything from older Permian to Precambrian rocks.

The 428-Ma isochron of Figure 15 suggests, as do the REE distributions, that the samples were largely unaffected by their diagenetic residence time in the evaporitic brines. This is important because it indicates that these clay mineral-oxyhydroxide assemblages may serve as getters for radioisotopes hypothetically released from breached containers of radioactive waste.

\section{Conclusions: Implications of the Geochemistry of the Clay Minerals and Insoluble Residues of WIPP Samples}

This report has emphasized primarily the importance of $\mathrm{Cl} / \mathrm{Br}, \mathrm{K} / \mathrm{Rb}, \mathrm{Th} / \mathrm{U}$ ratios, REE patterns, $\mathrm{K}-\mathrm{Ar}$ ages, and $\mathrm{Rb}-\mathrm{Sr}$ ages in assessing water/rock interactions in the horizons of the Salado Formation where the WIPP facility has been excavated. Although admittedly limited, the study allows the following observations to be made:

- The $x$-ray data indicate the presence of clay minerals and other insoluble phases normally expected in evaporites.

- $\mathrm{Cl} / \mathrm{Br}$ ratios for soluble material fall in the normal range for marine evaporites, although some samples are very low in total $\mathrm{Br}$.

- $\mathrm{Cl} / \mathrm{Br}$ ratios in insoluble material are very high, partly because of excess $\mathrm{Cl}$ from included halide minerals, and partly because of Br deficiency.

- The $\mathrm{K} / \mathrm{Rb}$ and $\mathrm{Th} / \mathrm{U}$ ratios for the insoluble material fall in the normal range for shales, suggesting that their internal alkali element content has been largely undisturbed by residence in the evaporites. Furthermore, there is no evidence to suggest that uranium has migrated from the samples, because the $\mathrm{Th} / \mathrm{U}$ ratios are fairly uniform and close to the values for shale. The overall Th and $U$ concentrations are somewhat lower than in shales. 
- The REE data for the insoluble material strongly suggest that the residence of these insoluble materials in the evaporite environment has not affected their incernal REE systematics. No evidence is noted for loss or gain of the REE in these insoluble materials.

- K-Ar dates for clay minerals range from $365 \mathrm{Ma}$ to $390 \mathrm{Ma}$, corresponding to the range for clay minerals from nearby sites (Broc':ins, 1981). These dates, well in excess of the evaporitic formational age of 220 to $230 \mathrm{Ma}$, suggest that residence in the evaporites has not significantly perturbed the $\mathrm{K}$-Ar systematics.

- WIPP-site clay minerals yield a $\mathrm{Rb}-\mathrm{Sr}$ isochron age of $428 \pm 7 \mathrm{Ma}$. This indicates little if any perturbation of $\mathrm{Rb}-\mathrm{Sr}$ systematics as a result of evaporite brineclay mineral reaction.

- The clay minerals and other insoluble materials appear resistant to destructive effects of their entrapment in the evaporites, suggesting that these insoluble materials would be good getters for radionuclides hypothetically released from radioactive wastes into the Salado Formation.

\section{References}

Anders, E., and M. Ebihara. 1982. "Solar-System Abundances of the Elements," Geochimica et Cosmochimica Acta. Vol. 45, no. 11, 2362-2380.

Brookins, D.G. 1979. Uranium Deposits of the Grants, New Mexico Mineral Belt II. Grand Junction, CO: US Department of Energy, Grand Junction Office.
Brookins, D.G. 1981. "Geochronologic Studies Near the WIPP Site, Southeastern New Mexico," Environmental Geology and Hydrology in New Mexico. Eds. S.G. Wells, W. Lambert, and J.F. Callender, New Mexico Geological Society Special Publication No. 10. Roswell, NM: New Mexico Geological Society. 147-152.

Brookins, D.G. 1982. "Study of Polyhalite From the WIPP Site, New Mexico," Scientific Basis for Nuclear Waste Management IV, Proceedings of the Materials Research Society Annual Meeting, Boston, MA, November 1981. Ed. S.V. Topp. New York, NY: North-Holland. Vol. 6, 257-264.

Brookins, D.G. 1983. "Eh-pH Diagrams for the Rare Earth Elements at $25{ }^{\circ} \mathrm{C}$ and One Bar Pressure," Geochemical Journal. Vol. 17, no. 5, 223-229.

Brookins, D.G. 1985. "Rubidium-Strontium Geochronologic Systematics in Igneous Contact Zones: Analog for Strontium-90 and Cesium-137 Behavior in the Near Field," Scientific Basis for Nuclear Waste Management VIII, Materials Research Society Symposia Proceedings, Boston, MA, November 26-29, 1984. Eds. C.M. Jantzen, J.A. Stone, and R.C. Ewing. Pittsburgh, PA: Materials Research Society. Vol. 44, 573-582.

Brookins, D.G, and S.J. Lambert. 1987a. "Radiometric Dating of Ochoan (Permian) Evaporites, WIPP Site, Delaware Basin, New Mexico, USA," Scientific Basis for Nuclear Waste Management X, Boston, MA, December 1-4, 1986. Eds. J.K. Bates and W.B. Seefeldt. SAND862192C. Pittsburgh, PA: Materials Research Society. Vol. 84, 771-780.

Brookins, D.G., and S.J. Lambert. 1987b. "K-Ar and $\mathrm{Rb}-\mathrm{Sr}$ Age Determinations from Clay Minerals and Related Minerals from the WIPP, Southeastern New Mexico," Isochron/West. No. 49, 4-7.

Brookins, D.G., and S.J. Lambert. 1988. "WIPP Site Studies: Secondary Selenite Veins in the Rustler Formation and Dewey Lake Red Beds," Scientific Basis for Nuclear Waste Management XI, Boston, MA, November 30-December 3, 1987. Eds. M.J. Apted and R.E. Westerman. SAND87-1610J. Pittsburgh, PA: Materials Research Society. Vol. 112, 233-241. 
Brookins, D.G., and J.K. Register. 1981. PotassiumArgon and Neutron Activation Analysis Studies of Polyhalites Near the WIPP Site. SAND81-7071. Albuquerque, NM: Sandia National Laboratories.

Brookins, D.G., J.K. Register, and H.W. Kreuger. 1980. "Potassium-Argon Dating of Polyhalite in Southeastern New Mexico," Geochimica et Cosmochimica Acta. Vol. 44, no. 5, 635-637.

Brookins, D.G., S.J. Lambert, and D.B. Ward. 1989. "Authigenic Clay Minerals in the Rustler Formation, WIPP Site Area, New Mexico," Scientific Basis for Nuclear Waste Management XIII, Boston, MA, November 27-December 2, 1989. Eds. V.M. Oversby and P.W. Brown. SAND89-1405C. Pittsburgh, PA: Materials Research Society. Vol. 176, 665-672.

Dean, W.E. 1978. "Trace and Minor Elements in Evaporites," Marine Evaporites, Lecture Notes for Short Course No. 4, Oklahoma City, OK, September 8, 1978. Eds. W.E. Dean and B.C. Schreiber. SEPM Short Course No. 4. Tulsa, OK: Society of Economic Paleontologists and Mineralogists. 86-104.

Elderfield, H., and M.J. Greaves. 1982. "The Rare Earth Elements in Seawater," Nature. Vol. 296, no. 5854, 214-219.

Haskin, L.A., and R.A. Schmitt. 1967. "Rare-Earth Distributions," Researches in Geochemistry. Ed. P.H. Abelson. New York, NY: John Wiley and Sons. Vol. 2, 234-258.

Minor, M.M., W.K. Hensley, M.M. Denton, and S.R. Garcia. 1981. An Automated Activation-Analysis System. LA-UR-81-1810. Los Alamos, NM: Los Alamos National Laboratory.

Register, J.K. 1981. Rubidium-Strontium and Related Studies of the Salado Formation, Southeastern New Mexico. SAND81-7072. Albuquerque, NM: Sandia National Laboratories.

Register, J.K., and D.G. Brookins. 1980. "Rb-Sr Isochron Age of Evaporite Minerals From the Salado Formation (Late Permian), Southeastern New Mexico," Isochron/West. No. 29, 39-42.
York, D. $1969 . \quad$ "Least-Squares Fitting of a Straight Line With Correlated Errors," Earth and Planetary Science Letters. Vol. 5, no. 5, 320-324. 


\section{DISTRIBUTION}

\section{Federal Agencies}

US Department of Energy (6)

Office of Civilian Radioactive Waste Management

Attn: Deputy Director, RW-2

Associate Director, RW-10/50

Office of Program and

Resources Management Office of Contract Business Management

Director, Analysis and

Verification Division, RW-22

Associate Director, RW-30

Office of Systems and

Compliance

Associate Director, RW-40

Office of Storage and

Transportation

Director, RW-4/5

Office of Strategic Planning and International Programs office of External Relations

Forrestal Building

Washington, DC 20585

US Department of Energy

Albuquerque Operations office

Attn: National Atomic Museum Library PO Box 5400

Albuquerque, NM 87185-5400

US Department of Energy (4)

WIPP Project Integration office

Attn: W.J. Arthur III

L.W. Gage

P.J. Higgins

PO Box 5400

D.A. Olona

Albuquerque, NM 87115-5400

US Department of Energy (2)

WIPP Project Integration Sate11ite Office

Attn: R. Batra

R. Becker

PO Box 3090, Mail Stop 525

Carlsbad, NM 88221-3090

US Department of Energy (3)

WIPP Project Site office (Carlsbad)

Attn: V. Daub

J. Lippis

J.A. Mewhinney

PO Box 3090

Carlsbad, NM 88221-3090
US Department of Energy

Research \& Waste Management Division Attn: Director

PO Box E

Oak Ridge, TN 37831

US Department of Energy

Attn: E. Young

Room E-178

GAO/RCED/GTN

Washington, DC 20545

US Department of Energy

Office of Environmental Restoration

and Waste Management

Attn: J. Lytle, EM-30, Trevion II

Washington, DC 20585-0002

US Department of Energy (3)

Office of Environmental Restoration and Waste Management

Attn: M. Frei, EM-34, Trevion II

Washington, DC 20585-0002

US Department of Energy

Office of Environmental Restoration and Waste Management

Attn: S. Schneider, EM-342, Trevion II

Washington, DC 20585-0002

US Department of Energy (2)

Office of Environment, Safety and Health

Attn: C. Borgstrom, EH-25

R. Pelletier, EH-231

Washington, DC 20585

US Department of Energy

Idaho Operations office

Fuel Processing and Waste

Management Division

785 DOE Place

Idaho Falls, ID 83402

US Environmental Protection Agency (2)

Radiation Protection Programs

Attn: $M$. Oge

ANR -460

Washington, DC 20460 
US Geological Survey (2)

Water Resources Division

Attn: R. Livingston

4501 Indian School NE

Suite 200

Albuquerque, NM 87110

US Nuclear Regulatory Commission Division of Waste Management

Attn: H. Marson

Mail Stop 4-H-3

Washington, DC 20555

Boards

Defense Nuclear Facilities Safety Board

Attn: D. Winters

625 Indiana Ave. NW, Suite 700

Washington, DC 20004

Nuclear Waste Technical Review Board (2)

Attn: Chairman S.J.S. Parry

1100 Wilson Blvd., Suite 910

Arlington, VA 22209-2297

Advisory Committee on Nuclear Waste

Nuclear Regulatory Commission

Attn: R. Major

7920 Norfolk Ave.

Bethesda, MD 20814

\section{State Agencies}

Environmental Evaluation Group (3) Attn: Library

7007 Wyoming NE

Suite F-2

Albuquerque, NM 87109

NM Bureau of Mines and Mineral Resources

Socorro, NM 87801

NM Energy, Minerals, and Natural Resources Department

Attn: Library

2040 S. Pacheco

Santa Fe, NM 87505

NM Environment Department (3)

Secretary of the Environment

Attn: J. Espinosa

1190 St. Francis Drive

Santa Fe, NM 87503-0968
NM Environment Department

WIPP Project Site

Attn: P. McCasland

PO Box 3090

Car1sbad, NM 88221

\section{Laboratories/Corporations}

Battelle Pacific Northwest

Laboratories

Attn: R.E. Westerman, MSIN P8-44

Battelle Blvd.

Richland, WA 99352

INTERA Inc.

Attn: J.F. Pickens

6850 Austin Center Blvd.

Suite 300

Austin, TX 78731

INTERA InC.

Attn: W. Stensrud

PO Box 2123

Carlsbad, NM 88221

IT Corporation

Attn: R.F. Mckinney

Regional office

5301 Central NE, Suite 700

Albuquerque, NM 87108

Lawrence Berkeley Laboratory

Earth Sciences Division

Att: $H$. Wollenberg

1 Cyclotron Rd.

Berkeley, CA 94720

Los Alamos National Laboratory

Attn: B. Erdal, CNC-11

PO Box 1663

Los Alamos, NM 87544

$\mathrm{RE} / \mathrm{SPEC}$, Inc.

Attn: W. Coons

4775 Indian School NE

Suite 300

Albuquerque, NM 87110-3927

$\mathrm{RE} / \mathrm{SPEC}$, Inc .

Attn: J.L. Ratigan

PO Box 725

Rapid City, SD 57709

Southwest Research Institute (2)

Center for Nuclear Waste

Regulatory Analysis

Attn: P.K. Nair

6220 Culebra Road

San Antonio, TX 78228-0510 
SAIC

Attn: D.C. Royer

101 Convention Center Dr.

Las Vegas, NV 89109

SAIC

Attn: H.R. Pratt

10260 Campus Point Dr.

San Diego, CA 92121

SAIC (2)

Attn: M. Davis

J. Tollison

2109 Air Park Rd. SE

Albuquerque, NM 87106

Tech Reps Inc. (3)

Attn: J. Chapman

C. Crawford

T. Peterson

5000 Marble NE, Suite 222

Albuquerque, NM 87110

TRW Environmental Safety Systems Attn: L. Wildman

2650 Park Tower Dr., Suite 1300

Vienna, VA 22180-7306

Westinghouse Electric Corporation (5)

Attn: Library

C. Cox

L. Fitch

B.A. Howard

PO Box 2078

R. Kehrman

Carlsbad, NM 88221

Westinghouse-Savannah River

Technology Center (4)

Attn: N. Bibler

J.R. Harbour

M.J. Plodinec

G.G. Wicks

Aiken, SC 29802

\section{National Academy of Sciences, WIPP Panel}

Howard Adler

Oak Ridge Associated Universities

Medical Sciences Division

PO Box 117

Oak Ridge, TN 37831-0117

Ina Alterman

Board on Radioactive

Waste Management, GF456

2101 Constitution Ave.

Washington, DC 20418
Fred M. Ernsberger

250 Old Mill Road

Pittsburgh, PA 15238

John D. Bredehoeft

Western Region Hydrologist

Water Resources Division

US Geological Survey (M/S 439)

345 Middlefield Road

Menlo Park, CA 94025

Rodney C. Ewing

Department of Geology

University of New Mexico

Albuquerque, NM 87131

Charles Fairhurst, Chairman

Department of Civil and

Mineral Engineering

University of Minnesota

500 Pillsbury Dr. SE

Minneapolis, MN 55455-0220

B. John Garrick

PLG Incorporated

4590 MacArthur Blvd., Suite 400

Newport Beach, CA 92660-2027

Leonard F. Konikow

US Geological Survey

431 National Center

Reston, VA 22092

Peter B. Myers

National Academy of Sciences

Board on Radioactive

Waste Management

2101 Constitution Ave.

Washington, DC 20418

Jeremiah O'Driscol1

Jody Incorporated

505 Valley Hill Drive

Atlanta, GA 30350

Christopher G. Whipple

Clement International

160 Spear St., Suite 1380

San Francisco, CA 94105

\section{Individuals}

P. Drez

8816 Cherry Hills Rd. NE Albuquerque, NM 87111 
D.W. Powers

Star Route Box 87

Anthony, TX 79821

\section{Universities}

University of New Mexico

Geology Department

Attn: Library

Albuquerque, NM 87131

University of Washington

College of Ocean \& Fishery Sciences

Attn: G.R.Heath

583 Henderson Hall

Seattle, WA 98195

\section{Libraries}

Thomas Brannigan Library

Attn: D. Dresp

106 W. Hadley St.

Las Cruces, NM 88001

Government Publications Department

Zimmerman Library

University of New Mexico

Albuquerque, NM 87131

Hobbs Public Library

Attn: M. Lewis

509 N. Ship St.

Hobbs, NM 88248

New Mexico Junior College

Panne11 Library

Attn: R. Hill

Lovington Highway

Hobbs, NM 88240

New Mexico State Library

Attn: N. McCallan

325 Don Gaspar

Santa Fe, NM 87503

New Mexico Tech

Martin Speere Memorial Library

Campus Street

Socorro, NM 87810

WIPP Public Reading Room

Carlsbad Public Library

Attn: Director

$101 \mathrm{~S}$. Halagueno St.

Carlsbad, NM 88220

\section{Foreign Addresses}

Studiecentrum Voor Kernenergie

Centre D'Energie Nucleaire

Attn: A. Bonne

SCK/CEN Boeretang 200

B-2400 Mo1, BELGIUM

Atomic Energy of Canada, Ltd.

Whiteshell Research Estab.

Attn: B. Goodwin

M. Stevens

D. Wushke

Pinewa, Manitoba, CANADA ROE 1LO

Francois Chenevier (2)

ANDRA

Route du Panorama Robert Schumann

B.P. 38

92266 Fontenay-aux-Roses, Cedex

FRANCE

Jean-Pierre Olivier

OECD Nuclear Energy Agency

Division of Radiation Protection and Waste Management

38, Boulevard Suchet

75016 Paris, FRANCE

Claude Sombret

Centre D'Etudes Nucleaires

De La Vallee Rhone

CEN/VALRHO

S.D.H.A. B.P. 171

30205 Bagnols-Sur-Ceze, FRANCE

Gesellschaft fur Reaktorsicherheit (GRS) (2)

Attn: B. Baltes

W. Muller

Schwertnergasse 1

D-5000 Cologne, GERMANY

Bundesanstalt fur Geowissenschaften und Rohstoffe

Attn: M. Langer

Postfach 510153

3000 Hanover 51, GERMANY

Bundesministerium fur Forschung und

Technologie

Postfach 200706

5300 Bonn 2, GERMANY 
Institut fur Tieflagerung (2)

Attn: K. Kuhn

Theodor-Heuss-Strasse 4

D-3300 Braunschweig, GERMANY

Physikalisch-Technische Bundesanstalt

Attn: P. Brenneke

Postfach 3345

D-3300 Braunschweig, GERMANY

Shingo Tashiro

Japan Atomic Energy Research Inst.

Tokai-Mura, Ibaraki-Ken, 319-11

JAPAN

Netherlands Energy Research

Foundation ECN

Attn: L.H. Vons

3 Westerduinweg

PO Box 1

1755 ZG Petten, THE NETHERLANDS

Svensk Karnbransleforsorjning $A B$

Attn: F. Karlsson

Project KBS

Karnbrans lesakerhet

Box 5864

10248 Stockholm, SWEDEN

Nationale Genossenschaft für die

Lagerung radioaktiver Abfälle (2)

Attn: S. Vomvoris

P. Zuidema

Hardstrasse 73

CH-5430 Wettingen, SWITZERLAND

AEA Technology

Attn: J.H. Rees

D5W/29 Culham Laboratory

Abington, Oxfordshire OX14 3DB

UNITED KINGDOM

AEA Technology

Attn: W.R. Rodwell

044/A31 Winfrith Technical Centre

Dorchester, Dorset DT2 8DH

UNITED KINGDOM

AEA Technology

Attn: J.E. Tinson

B4244 Harwel1 Laboratory

Didcot, Oxfordshire OX11 ORA

UNITED KINGDOM
D.R. Knowles

British Nuclear Fuels, plc

Risley, Warrington, Cheshire WA3 6AS

1002607 UNITED KINGDOM

\section{Internal}

$\begin{array}{ll}1502 & \text { J.C. Cummings } \\ 6000 & \text { D.L. Hartley } \\ 6118 & \text { S.J. Lambert (5) } \\ 6119 & \text { E.D. Gorham } \\ 6119 & \text { Staff (14) } \\ 6121 & \text { J.R. Tillerson } \\ 6121 & \text { Staff (7) } \\ 6300 & \text { D.E. Ellis } \\ 6302 & \text { L.E. Shephard } \\ 6303 & \text { S.Y. Pickering } \\ 6303 & \text { W.D. Weart } \\ 6305 & \text { S.A. Goldstein } \\ 6306 & \text { A.L. Stevens } \\ 6342 & \text { D.R. Anderson } \\ 6342 & \text { Staff (20) } \\ 6343 & \text { V. Harper-Slaboszewicz } \\ 6343 & \text { Staff (2) } \\ 6345 & \text { R.C. Lincoln } \\ 6345 & \text { Staff (9) } \\ 6347 & \text { D.R. Schafer } \\ 6348 & \text { J.T. Holmes } \\ 6348 & \text { Staff (4) } \\ 6351 & \text { R.E. Thompson } \\ 6352 & \text { D.P. Garber } \\ 6352 & \text { S.E. Sharpton } \\ 6352 & \text { WIPP Central Files (10) } \\ 7141 & \text { Technical Library (5) } \\ 7151 & \text { Technical Publications } \\ 7613-2 & \text { Document Processing for } \\ & \text { DoE/OSTI (10) } \\ 8523-2 & \text { Central Technical Files }\end{array}$



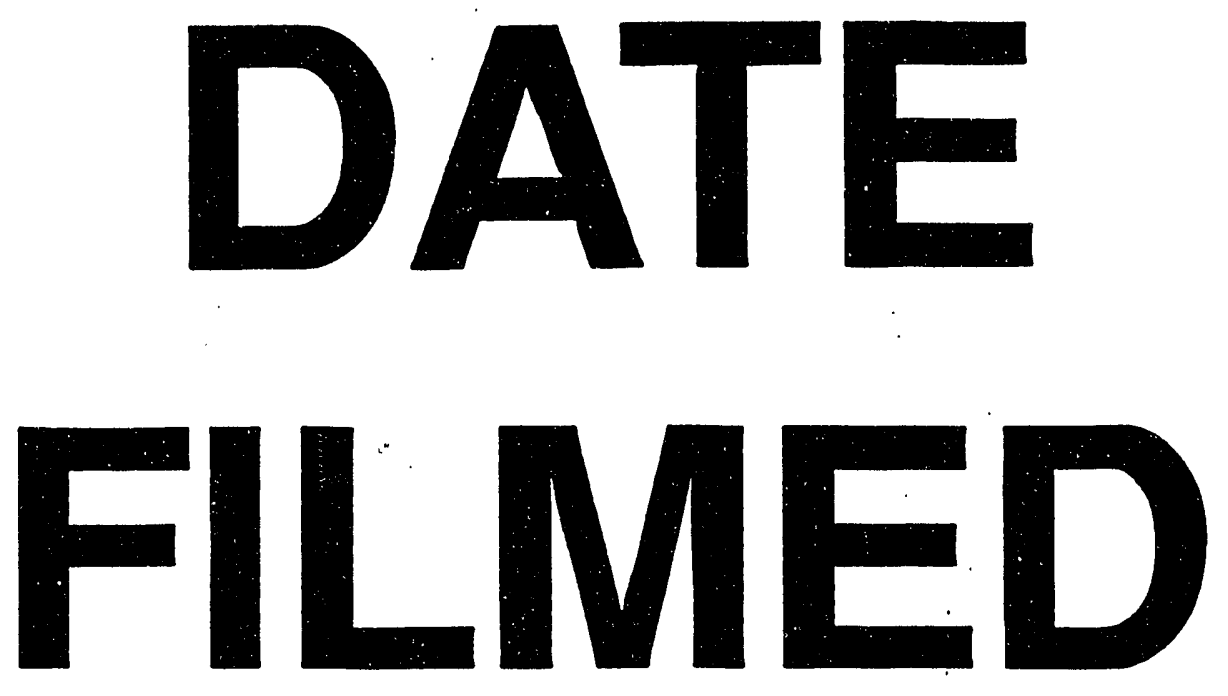

$9 / 29 / 93$
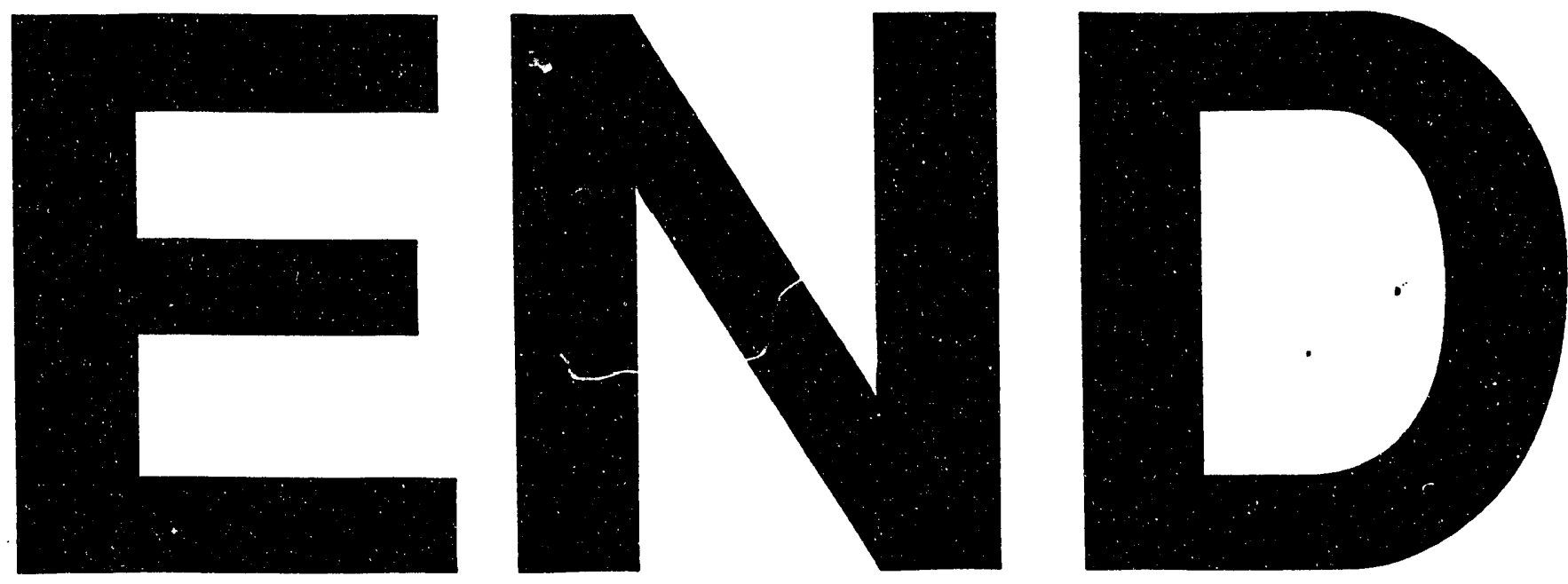
